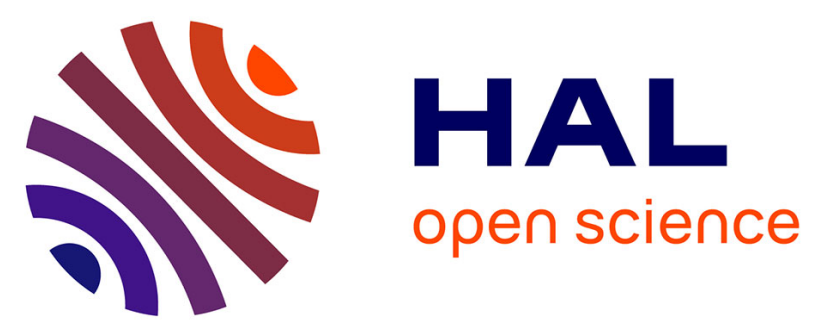

\title{
Charge-density analysis using multipolar atom and spherical charge models: \\ 2-methyl-1,3-cyclopentanedione, a compound displaying a resonance-assisted hydrogen bond
}

Ayoub Nassour, Maciej Kubicki, Jonathan Wright, Teresa Borowiak, Grzegorz Dutkiewicz, Claude Lecomte, Christian Jelsch

\section{To cite this version:}

Ayoub Nassour, Maciej Kubicki, Jonathan Wright, Teresa Borowiak, Grzegorz Dutkiewicz, et al.. Charge-density analysis using multipolar atom and spherical charge models: 2-methyl-1,3cyclopentanedione, a compound displaying a resonance-assisted hydrogen bond. Acta Crystallographica Section B : Structural Science, Crystal Engineering and Materials [2014-..], 2014, 70 (2), pp.197-211. 10.1107/S2052520613031375 . hal-02365152

\section{HAL Id: hal-02365152 \\ https://hal.science/hal-02365152}

Submitted on 15 Nov 2019

HAL is a multi-disciplinary open access archive for the deposit and dissemination of scientific research documents, whether they are published or not. The documents may come from teaching and research institutions in France or abroad, or from public or private research centers.
L'archive ouverte pluridisciplinaire HAL, est destinée au dépôt et à la diffusion de documents scientifiques de niveau recherche, publiés ou non, émanant des établissements d'enseignement et de recherche français ou étrangers, des laboratoires publics ou privés. 


\section{Charge-density analysis using multipolar atom and spherical charge models: 2-methyl-1,3-cyclopentanedione, a compound displaying a resonance-assisted hydrogen bond}

\section{Ayoub Nassour, Maciej Kubicki, Jonathan Wright, Teresa Borowiak, Grzegorz Dutkiewicz, Claude Lecomte and Christian Jelsch}

Acta Cryst. (2014). B70, 197-211

Copyright (C) International Union of Crystallography

Author(s) of this paper may load this reprint on their own web site or institutional repository provided that this cover page is retained. Republication of this article or its storage in electronic databases other than as specified above is not permitted without prior permission in writing from the IUCr.

For further information see http://journals.iucr.org/services/authorrights.html

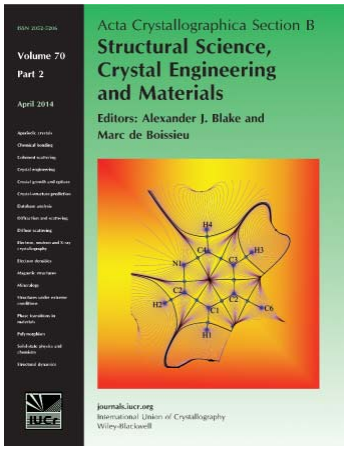

Acta Crystallographica Section B: Structural Science, Crystal Engineering and Materials publishes scientific articles related to the structural science of compounds and materials in the widest sense. Knowledge of the arrangements of atoms, including their temporal variations and dependencies on temperature and pressure, is often the key to understanding physical and chemical phenomena and is crucial for the design of new materials and supramolecular devices. Acta Crystallographica $B$ is the forum for the publication of such contributions. Scientific developments based on experimental studies as well as those based on theoretical approaches, including crystal-structure prediction, structureproperty relations and the use of databases of crystal structures, are published.

Crystallography Journals Online is available from journals.iucr.org 
Acta Crystallographica Section B

Structural Science, Crystal Engineering and Materials

ISSN 2052-5206

\section{Ayoub Nassour, ${ }^{a}$ Maciej Kubicki, ${ }^{\text {b Jonathan Wright, }}{ }^{\text {c }}$ Teresa Borowiak, ${ }^{b}$ Grzegorz Dutkiewicz, ${ }^{b}$ Claude Lecomte ${ }^{a}$ and Christian Jelsch ${ }^{a_{*}}$}

a'Laboratoire de Cristallographie, Résonance Magnétique et Modélisations, CNRS UMR 7036, Université de Lorraine, BP 70239, 54506 Vandoeuvre-les-Nancy CEDEX, France, ${ }^{\mathbf{b}}$ Faculty of Chemistry, Adam Mickiewicz University, Grunwaldzka 6, 60-780 Poznań, Poland, and 'European Synchrotron Radiation Facility (ESRF), 6 Rue Jules Horowitz, BP 220, 38043 Grenoble CEDEX 9, France

Correspondence e-mail:

christian.jelsch@univ-lorraine.fr

\section{Charge-density analysis using multipolar atom and spherical charge models: 2-methyl-1,3-cyclopent- anedione, a compound displaying a resonance- assisted hydrogen bond}

The experimental charge-density distribution in 2-methyl-1,3cyclopentanedione in the crystal state was analyzed by synchrotron X-ray diffraction data collection at $0.33 \AA$ resolution. The molecule in the crystal is in the enol form. The experimental electron density was refined using the Hansen-Coppens multipolar model and an alternative modeling, based on spherical atoms and additional charges on the covalent bonds and electron lone-pair sites. The crystallographic refinements, charge-density distributions, molecular electrostatic potentials, dipole moments and intermolecular interaction energies obtained from the different charge-density models were compared. The experimental results are also compared with the theoretical charge densities using theoretical structure factors obtained from periodic quantum calculations at the B3LYP/6-31G** level. A strong intermolecular $\mathrm{O}-\mathrm{H} \cdots \mathrm{O}$ hydrogen bond connects molecules along the [001] direction. The deformation density maps show the resonance within the $\mathrm{O}=\mathrm{C}-\mathrm{C}=\mathrm{C}-\mathrm{OH}$ fragment and merged lone pair lobes on the hydroxyl $\mathrm{O}$ atom. This resonance is further confirmed by the analysis of charges and topology of the electron density.

\section{Introduction}

Cyclic $\beta$-diketoalkanes exist in the crystalline state in the enol form. They tend to form molecular aggregates (usually $\beta$ chains) connected by strong $\mathrm{O}-\mathrm{H}$. . O hydrogen bonds with distances between their hydroxyl and carbonyl $\mathrm{O}$ atoms often shorter than $2.6 \AA$. The stereochemical configuration of these hydrogen bonds can be conveniently described using the syn/ anti - SYN/ANTI nomenclature proposed by Etter et al. (1986). The descriptors syn and anti are related to the positions of the $\mathrm{H}$ atom and carbonyl lone pairs with respect to the bonds to the $\mathrm{C} 2$ atom. Lower and upper cases are related to the hydroxyl and carbonyl groups, respectively.

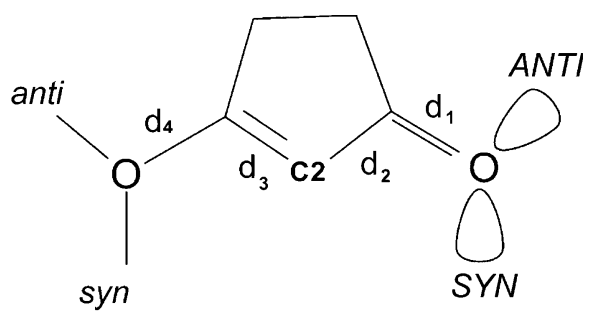

All possible hydrogen-bond configurations were found in the crystal structures of $\beta$-diketoalkanes. For example, the syn/ $S Y N$ configuration exists in dimedone (5,5-dimethyl-1,3cyclohexanedione; Semmingsen, 1974; Singh \& Calvo, 1975), anti/ANTI in 1,3-cyclohexanedione (Etter et al., 1986) and 1,3cyclopentanedione (Katrusiak, 1990a), syn/ANTI in 6:1 1,3cyclohexanedione:benzene inclusion crystals (Etter et al.,
Received 26 August 2013

Accepted 15 November 2013 
1986) and anti/SYN in squaric acid (3,4-dihydroxy-3-cyclobutene-1,2-dione; Semmingsen et al., 1977).

The room-temperature medium-resolution crystal structure of the title compound, 2-methyl-1,3-cyclopentanedione (hereinafter referred to as MCPD) was determined by Katrusiak (1989). That study confirmed the enol form of the compound, and the molecule was found to lie on a crystallographic mirror plane in the space group $C 2 / \mathrm{m}$. It was also suggested that the methyl group is slightly disordered. In the main configuration, the $\mathrm{H}$ atom that lies in the mirror plane is on the hydroxyl side while the metastable position corresponds to a $180^{\circ}$ rotation of the methyl group (i.e. the $\mathrm{H}$ atom in a special position is on the carbonyl side).

MCPD crystals exhibit exceptional stability. Unlike closely related 1,3-cyclopentanedione (CPD) and 1,3-cyclohexanedione (CHD), they do not undergo any pressure-induced phase transition up to 3.01 (5) GPa (Katrusiak, 1991). The phase transition in CHD (anti/ANTI configuration) takes place below $0.3 \mathrm{GPa}$ and is caused by collective transitions of enolic protons accompanied by the inversion of the sites of hydroxyl and carbonyl groups (Katrusiak, 1990b). Crystals of CPD presumably undergo a similar phase transition, but during this transition, crystals shatter into small pieces (Katrusiak, 1990b). The stability of MCPD crystals was explained on a structural basis by the energetically favourable orientation of the dipole moments of the molecules belonging to neighbouring hydrogen-bonded chains. Taking into account the proposed mechanism of the phase transition, the stability of MCPD may also be related to the more localized electronic structure of this compound, which makes the proton transfer less probable.

$\beta$-Diketone enols were used as one of the model compounds for a concept of so-called resonance-assisted hydrogen bonds (RAHB), introduced by Gilli and co-workers (Gilli et al., 1989). This idea was developed in order to explain the existence of strong intra- and intermolecular hydrogen bonds in which nominally no charges are involved, in contrast to the majority of strong hydrogen bonds. RAHB (or $\pi$ cooperative hydrogen bond: Jeffrey \& Saenger, 1991) was proposed as a kind of feedback mechanism of hydrogen-bond strengthening accompanied by an increase of $\pi$-electron delocalization within the resonance fragment. It was successfully applied to a wide range of intra- $[$ e.g. $\beta$-diketone enols (Gilli et al., 1989; Bertolasi et al., 1991), 1,3-diketone arylhydrazones (Bertolasi et al., 1993), $\beta$-enaminones (Gilli et al., 2000)] and intermolecular hydrogen bonds [e.g. $\beta$-diketone enols (Gilli et al., 1993), anti- $\beta$-ketoarylhydrazones (Bertolasi, Gilli, Ferretti, Gilli, Vaughan \& Jollimore, 1999), NH-pyrazoles (Bertolasi, Gilli, Ferretti, Gilli \& Fernàndez-Castaño, 1999), secondary enaminones (Bertolasi et al., 1998)]. The compound for which RAHB can be postulated should contain $D-\mathrm{H}$ and $A=X(D, A-$ hydrogen bond donor and acceptor, respectively), connected by a spacer capable of forming a resonance path. An experimental charge-density analysis of benzoylacetone (8.4 K X-ray data and $20 \mathrm{~K}$ neutron data; Madsen et al., 1998) followed by ab initio calculations (Schiøtt et al., 1998) showed the extensive $\pi$-delocalization in the keto- enol groups, and a strong, slightly asymmetric intramolecular $\mathrm{O}$... H. . O hydrogen bond. The results of these studies led to a modification of the original idea of Gilli et al. (1989), according to which the RAHB may be regarded as a kind of synergetic mechanism that acts towards maintaining zero partial charges on the two $\mathrm{O}$ atoms. Hence, Madsen et al. (1998) showed that there are substantial negative partial charges on both $\mathrm{O}$ atoms accompanied by the positive charge on the $\mathrm{H}$ atom. Madsen et al. (1998) proposed, therefore, that RAHB is indeed a feedback mechanism but it changes the charges in hydrogen bonds towards a symmetrical distribution. For strong intramolecular hydrogen bonds, the resonanceinduced charges can compensate by pushing the $\mathrm{H}$ atom farther from the enol $\mathrm{O}$ atom, but also by transferring the electrons from the hydrogen onto this $\mathrm{O}$ atom.

The independent atom model (IAM) that uses spherical scattering factors does not allow a detailed description of chemical bonding and a deep understanding of the chemistry of a given compound. The experimental charge-density analysis of MCPD was performed to determine the distribution of the bonding density within the conjugated $\mathrm{O}=\mathrm{C}-$ $\mathrm{C}=\mathrm{C}-\mathrm{OH}$ fragment. The crystallographic refinement with two different models of the electron density were compared: the multipolar atom model (Hansen \& Coppens, 1978) and a model based on spherical atoms and additional charges on the expected sites of bonding density and electron lone pairs. The charge-density analysis issued from synchrotron experimental diffraction data was complemented by using structure factors obtained from first principles calculations in the crystalline state. Different molecular properties such as the electrostatic potential, interaction energy, dipole moment derived from the two models are compared.

\section{Materials and methods}

\subsection{Crystal data collection}

MCPD crystals, recrystallized from ethanol, were kindly provided by Professor Andrzej Katrusiak, Department of Chemistry, Adam Mickiewicz University, Poznań, Poland. A colourless single crystal of dimensions $\sim 0.1 \times 0.2 \times 0.3 \mathrm{~mm}$ was selected and mounted in a MiTeGen cryoloop.

The data collection was performed on ESRF beamline ID11 at a short wavelength of $\lambda=0.21784 \AA$ radiation and a zero $2 \theta$ angle for the two-dimensional detector. There was a single vertical rotation axis and the vertical beam size was varied to give different spots in the dynamic range of the detector. The beam size was $\sim 5 \mu \mathrm{m}$ for strong, $50 \mu \mathrm{m}$ for medium and $300 \mu \mathrm{m}$ for weak reflections. 11 scans were merged into a unique reflections file. An Oxford Instruments Cryosystem cooling device maintained the temperature at $100 \mathrm{~K}$ during the experiment.

The runs were divided into five sets with different distances of the CCD detector centre. The majority of the data were collected with a short sample-to-detector distance to maximize the data resolution. In order to fill in the low-resolution data, a far distance was also used. A series of exposure times were 
Table 1

Experimental details.

\begin{tabular}{ll}
\hline Crystal data & \\
Chemical formula & $\mathrm{C}_{6} \mathrm{H}_{8} \mathrm{O}_{2}$ \\
$M_{\mathrm{r}}$ & 112.13 \\
Crystal system, space group & Monoclinic, $C 2 / m$ \\
$a, b, c(\AA)$ & $12.402(3), 6.470(2), 6.321(2)$ \\
$\beta\left({ }^{\circ}\right)$ & $93.69(2)$ \\
$Z$ & 4 \\
Radiation type & Synchrotron, $\lambda=0.21784 \AA$ \\
$\mu\left(\mathrm{mm}^{-1}\right)$ & 0.001 \\
Crystal size $(\mathrm{mm})$ & $0.3 \times 0.2 \times 0.1$ \\
& \\
Data collection & \\
Diffractometer & Huber, ID11/ESRF \\
Absorption correction & - \\
No. of measured, independent and & $258713,7435,7319$ \\
$\quad$ observed $[I>2.0 \sigma(I)]$ reflections & \\
$R_{\text {int }}$ & 0.049 \\
$(\text { sin } \theta / \lambda)_{\text {max }}\left(\AA^{-1}\right)$ & 1.516 \\
Average redundancy & 24.7 \\
& \\
Refinement & \\
$R\left[F^{2}>2 \sigma\left(F^{2}\right)\right], w R\left(F^{2}\right), S$ & $0.034,0.031,1.05$ \\
No. of reflections & 7434 \\
No. of parameters & 186 \\
No. of restraints & 15 \\
H-atom treatment & Only H-atom coordinates refined \\
$\Delta \rho_{\text {max }}, \Delta \rho_{\text {min }}\left(\mathrm{e} \AA^{-3}\right)$ & $0.55,-0.36$ \\
\hline
\end{tabular}

Computer programs: MoPro (Jelsch et al., 2005); MoProViewer (Guillot, 2011).

used to maximize the dynamic range for data collection, which is otherwise limited by the 16-bit CCD Frelon4M camera. The crystal-to-detector distance was $15.68 \mathrm{~cm}$ with $1 \mathrm{~s}$ exposure time and $6.85 \mathrm{~cm}$ with 1 or $3 \mathrm{~s}$ exposure time. Data up to $\sin \theta / \lambda=1.51 \AA^{-1}$ were collected using the $\omega$-scan method with a $1^{\circ}$ rotation per frame. Crystallographic data and details of the data collection are given in Table 1.

Integration of reflection intensities, data reduction, Lorentz-polarization corrections and data merging were carried out with $S A D A B S$ and $S A I N T$ software (Bruker, 2004). The absorption coefficient $\mu=0.001 \mathrm{~mm}^{-1}$ was found to be negligible. The reflection intensities were corrected using experimentally determined factors as a function of incident angle for incomplete absorption of high-energy X-rays on the CCD detector (Wu et al., 2002). The measured reflections were merged into 7434 unique data, which corresponds to $98 \%$ completeness to the reciprocal resolution $s=1.51 \AA^{-1}$. Such a high resolution is not common and the average $I_{\mathrm{obs}} / \sigma\left(I_{\mathrm{obs}}\right)$ is still above 20 in the highest resolution shell (Fig. S3 of the supporting information $\left.{ }^{\mathbf{1}}\right)$. At very high resolution, the average ratio $\left\langle F_{\text {obs }}\right\rangle /\left\langle F_{\text {calc }}\right\rangle$ (Zhurov et al., 2008; Fig. S3) increases to values reaching 1.1, indicating a possible scale factor problem for these data.

\subsection{Multipolar experimental refinement (EXP_MUL)}

The crystal structure was solved by direct methods with SHELXS97 (Sheldrick, 2008) and refined initially by full-

\footnotetext{
${ }^{1}$ Supporting information for this paper is available from the IUCr electronic archives (Reference: PI5017).
}

matrix least-squares using SHELXL97 (Sheldrick, 2008). All $\mathrm{H}$ atoms were located in the difference Fourier map.

The charge density was subsequently refined against structure factors using the program MoPro (Guillot, Viry et al., 2001; Jelsch et al., 2005). The program includes stereochemical and dynamical restraints as well as the multipolar scattering factor formalism of Hansen \& Coppens (1978).

The multipolar expansion was terminated at the hexadecapolar level for $\mathrm{C}$ and $\mathrm{O}$ atoms and at a bond-directed dipole for hydrogen. The core and valence spherical scattering factors were calculated from Su \& Coppens (1998), wavefunctions for isolated atoms and the anomalous dispersion coefficients were taken from Kissel et al. (1995).

The values of the $U^{i j}$ parameters for $\mathrm{H}$ atoms were fixed, as taken from the SHADE server (Madsen, 2006). The $\mathrm{H}-X$ distances of $\mathrm{H}$ atoms were restrained to the values obtained from neutron diffraction studies (Allen, 1986) with a restraint sigma of $0.002 \AA$.

The O3-H3 ‥ 1 hydrogen bonds form an angle of $169.0^{\circ}$. As this angle is larger than $160^{\circ}$, the formula proposed by Yukhnevich (2009), which links the $d_{\mathrm{OH}}$ and $d_{\mathrm{O}} \ldots \mathrm{H}$ distances, can be applied. The distance $d_{\mathrm{H} 3 \ldots \mathrm{O} 1}=1.0212 \AA$ computed this way was used as a restraint target with a smaller sigma of $0.0002 \AA$. The application of the $d_{\mathrm{H} 3} \ldots$ O 1 restraint was deemed necessary, as its removal leads to non-realistic charge density on the hydroxyl group.

The charge density was subsequently refined against structure-factor amplitudes using the multipolar Hansen-Coppens model (Hansen \& Coppens, 1978) for pseudoatom electron density

$$
\begin{aligned}
\rho_{\text {atom }}(r)= & \rho_{\text {core }}(r)+P_{\text {val }} \kappa^{3} \rho_{\text {val }}(\kappa r) \\
& +\Sigma_{l} \kappa^{\prime 3} R_{l}\left(\kappa^{\prime} r\right) \Sigma_{m} P_{l m \pm} d_{l m \pm}(\theta, \varphi)
\end{aligned}
$$

where the first two terms are the spherically averaged core and valence electron densities of the atom, and the last term corresponds to the non-spherical valence density which is described in terms of real spherical harmonic functions. $P_{\text {val }}$ is the valence population, $P_{l m \pm}$ are the multipole populations and $\kappa$ and $\kappa^{\prime}$ are the contraction/expansion parameters. $R_{l}$ are radial Slater-type functions.

The refinement was performed against all the structurefactor amplitudes. Due to the exact $C_{s}$ symmetry of the MCPD molecule, the coordinate systems of all non-H atoms were chosen with two axes in the (010) molecular plane (crystallographic mirror) and one axis perpendicular to this plane (b). As a consequence, the $P_{l m}$ coefficients of the multipole functions antisymmetric with respect to the $\mathbf{b}$ axis are strictly zero values. With a reduced number of refined multipoles and the ultra-high resolution of the diffraction data, the $\mathrm{C}$ and $\mathrm{O}$ atoms could be modeled up to the hexadecapolar level.

Due to the very high resolution of the data measured using a synchrotron $\mathrm{X}$-ray beam, the vast majority of reflections are at high order: $87 \%$ at $d<0.7 \AA$ and $70 \%$ at $d<0.5 \AA$. Therefore, no-high order refinement was deemed necessary to refine the atomic positions and displacement parameters of non-H atoms. The reliability of the anisotropic displacement 
Table 2

Statistics on the crystallographic refinements versus the experimental and theoretical structure factors for the different atom models.

The $\Delta \rho_{\min }$ and $\Delta \rho_{\max }$ values of the residual density and the r.m.s. of $\Delta \rho$ are also computed using reflections up to $s=0.7 \AA^{-1}$

\begin{tabular}{lllll}
\hline Refinement data & MUL EXP & VIR EXP & MUL THEO & VIR THEO \\
\hline No. of unique reflections & 7434 & & 7580 & \\
No. of variables & 349 & 292 & 266 & 116 \\
$R(F)$ & 0.035 & 0.037 & 0.012 & 0.012 \\
$w R_{2}\left(F^{2}\right)$ & 0.031 & 0.033 & 0.011 & 0.012 \\
Goodness-of-fit $(F)$ & 1.03 & 1.12 & 0.10 & 0.12 \\
$\Delta \rho_{\max }, \Delta \rho_{\text {min }}$, r.m.s. & $+0.14,-0.15,0.033$ & $+0.16,-0.20,0.040$ & $+0.07,-0.13,0.013$ & $+0.10,-0.10,0.016$ \\
$\quad(\Delta \rho)\left(\mathrm{e} \mathrm{A}^{-3}\right)$ & & & & \\
\hline
\end{tabular}

to obtain an evaluation of the net atomic charges (Coppens et al., 1979).

\subsection{Multipolar theoretical refine- ment (THEO_MUL)}

The least-squares refinement versus theoretical structure factors was performed using all reflections up to $s=1.51 \AA^{-1}$. Compared with 7434 experimental data, only 156 reflections were not measured at that resolution. Refinement of the chargedensity parameters using the MoPro package (Jelsch et al., 2005) was performed versus the theoretical

parameters $U^{i j}$ was checked using the Hirshfeld (1976) rigidbond test. Differences between the components of $U^{i j}$ tensors along the non-H atoms bonds are all small, with a maximum of $6 \times 10^{-4} \AA^{2}$ for the $\mathrm{C} 21-\mathrm{C} 2$ bond.

All the different types of parameters were refined successively (scale factor, $X Y Z, U^{i j}, P_{\text {val }}, \kappa, P_{l m}, \kappa^{\prime}$ ). This whole procedure was recycled until convergence. For the $\mathrm{H}$ atoms, $\kappa^{\prime}$ values were restrained to $1.16(1)$ (Stewart, 1976). The $\kappa$ coefficients of the $\mathrm{C}$ and $\mathrm{O}$ atoms were restrained $\left(\sigma_{r}=0.003\right)$ to be correlated with the atomic charges $q=N_{\text {val }}-P_{\text {val }}$ (Volkov et al., 2001; Jelsch et al., 2005). The crystallographic statistics of the different refinements are given in Table 2.

\subsection{Theoretical structure factors}

Periodic quantum mechanical calculations using CRYSTAL09 (Dovesi et al., 2010) were performed at the crystal geometry observed experimentally and, using this as a starting geometry, optimization was performed with the density functional theory (DFT) method (Hohenberg \& Kohn, 1964) and with the B3LYP hybrid functional (Lee et al., 1988; Becke, 1993) using a 6-31G (d,p) basis set (Hariharan \& Pople, 1973). The level of accuracy in evaluating the Coulomb and exchange series is controlled by five parameters for which the values of $\left(I T O L_{\mathrm{i}}=6, i=1,4\right)$ and $I T O L_{5}=17$ were used for the Coulomb and exchange series. The shrinking factor of the reciprocal space was set to 4 , corresponding to $30 k$ points in the irreducible Brillouin zone at which the Hamiltonian matrix was diagonalized. Upon convergence on energy $(\Delta E \simeq$ $10^{-6}$ hartree), the periodic wavefunction based on the optimized geometry was obtained. The coordinates of $\mathrm{H}$ atoms were relaxed, but the unit cell was kept fixed. The index generation scheme proposed by Le Page \& Gabe (1979) was applied to generate 18616 unique Miller indices up to $s=$ $1.51 \AA^{-1}$ reciprocal resolutions. The option $X F A C$ of the CRYSTALO9 program was then used to generate a set of theoretical structure factors from the computed electron density and using a set of prepared indices. The theoretical charge density was refined versus all the generated structurefactors amplitudes.

A $P_{\mathrm{val}}-\kappa$ refinement was also performed, from the final multipolar model with all $P_{l m}$ coefficients set to zero, in order structure-factor amplitudes $F_{h k l}$. Multipoles were developed up to the hexadecapole level for the $\mathrm{O}$ and $\mathrm{C}$, and for the $\mathrm{H}$ atoms up to quadrupole level.

The multipolar atom refinement differs from the experimental refinement in the following manner:

(i) the atomic positions were kept fixed to the values obtained from the relaxation geometry;

(ii) the scale factor was fixed to the absolute value (1.0);

(iii) the atomic thermal motion parameters were set to zero;

(iv) restraints on the electron density distribution were imposed only on the $\mathrm{H}$ atoms. Their $\kappa$ parameters were restrained to a value of 1.16 (1) (Stewart, 1976).

\subsection{Virtual and real spherical atoms model}

Accurate electron density in crystals can be derived from aspherical corrections terms in the atomic model (Hirshfeld, 1976; Stewart, 1977). The 'multipolar' atom model, suggested by Hansen \& Coppens (1978), is now the most widely used in charge-density analysis. As shown before, this model describes the electron density as a sum of pseudo-atomic densities composed of a spherical and a multipolar part.

The electron density obtained from the multipolar model deviates from the spherical atom model mostly by an accumulation of electrons on the covalent bonds and on the lonepair regions. Based on these considerations, an empirical virtual and real spherical atom model that reproduces results of quality nearly comparable to the multipolar atom model was developed (Dadda et al., 2012). Such spherical charge modelling was already applied on several molecules in the past, notably on urea (Scheringer et al., 1978), diborane (Mullen \& Hellner, 1977) and silicon (Scheringer, 1980). More recently, the modelling of bond scatterers was applied by Afonine et al. $(2004,2007)$ in the refinement of proteins at ultra-high resolution.

In this model, the electron density is considered as a superposition of spherical real and virtual atoms

$$
\rho(r)=\sum_{\text {atoms }} \rho_{\text {core }}(r)+P_{\text {val }} \kappa^{3} \rho_{\text {val }}(\kappa r)+\sum_{\text {vir }} P_{\text {vir }} \kappa_{\text {vir }}^{3} \rho_{\text {vir }}(\kappa r)
$$


where $\rho_{\text {core }}$ and $\rho_{\text {val }}$ are the core and spherical valence electron densities that can be calculated from Hartree-Fock (HF) or density functional theory (DFT) methods. The real atoms $(\mathrm{C}$, $\mathrm{O}$ and $\mathrm{H}$ here) were treated spherically; these atoms can be described as the first and second terms of equation (1). The third term corresponds to the electron density $\rho_{\text {vir }}$ generated by the virtual atoms. The refined parameters here are a spherical valence population $P_{\text {vir }}$ and an expansion/contraction coefficient $\kappa_{\mathrm{vir}}$, in addition to the positions of the virtual atoms.

The same Slater-type wavefunction sum was adopted for the virtual atoms as in Dadda et al. (2012). The $Q_{\mathrm{Lp}}$ virtual atoms modelling the lone pairs were assigned the same wavefunction description as the $Q_{A B}$ bond scatterers, but, as they are more contracted in space, refined to larger $\kappa$ values.

To improve the convergence of the refinement, distances, distance similarity and planarity restraints/constraints were applied to the virtual atoms (Table S1). The $Q_{A B}$ virtual atoms were constrained to remain on the $A-B$ covalent bonds. The $Q_{\mathrm{Lp}}$ virtual atoms were stabilized by application of the distance $\left(\mathrm{O}-Q_{\mathrm{Lp}}\right)$ and angle similarity $\left(\mathrm{C}-\mathrm{O}-\mathrm{Q}_{\mathrm{Lp}}\right)$ restraints.

\subsection{Virtual atom theoretical refinement (THEO_VIR)}

The least-squares refinement versus theoretical structure factors were performed using all reflections up to $s=1.51 \AA^{-1}$. In the THEO_VIR model, only the charge-density parameters $P_{\mathrm{val}}, P_{\mathrm{vir}}, \kappa$ and $\kappa_{\mathrm{vir}}$, and the positions of virtual atoms were refined. The bond virtual atoms were initially placed on the

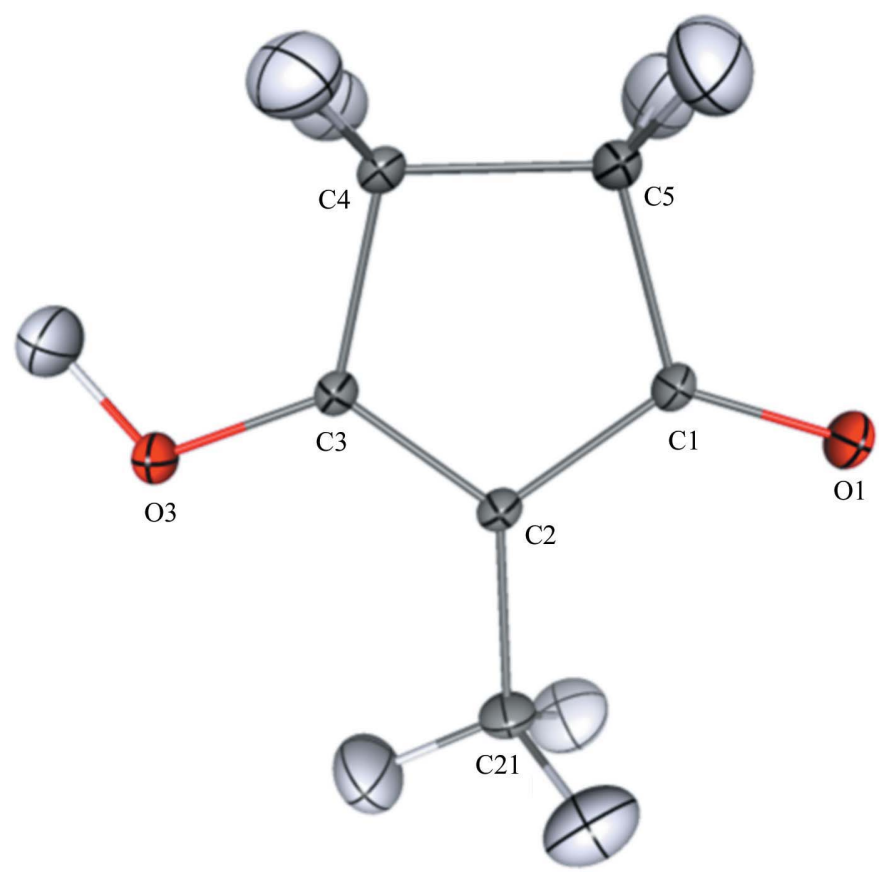

Figure 1

Diagram of the MCPD molecule with atom-labelling scheme. Displacement ellipsoids are shown at $50 \%$ probability level (those for $\mathrm{H}$ atoms are calculated with the SHADE server). The diagram was produced with MoProViewer (Guillot, 2011). middle of the covalent bonds; the electron LPs were placed at ideal distance restraint positions. $P_{\text {vir }}$ values were initially set to zero (which renders the virtual atoms devoid of electron density), therefore, the valence populations $P_{\text {val }}$ and $P_{\text {vir }}$ were the first parameters to be refined.

\subsection{Virtual atoms experimental refinement (EXP_VIR)}

The least-squares refinement based on experimental intensities $\left|F_{h k l}\right|^{2}$ was performed using all reflections up to $s=$ $1.52 \AA^{-1}$. The anisotropic $U^{i j}$ values of the bond virtual atoms were not refined, but constrained to take the average $U^{i j}$ values of the two bonding atoms. The $U^{i j}$ values of the $Q_{\mathrm{Lp}}$ virtual atoms were constrained to ride on the $U^{i j}$ values of the carrier atom. The different types of parameters (scale factor, $X Y Z, U^{i j}, P_{\text {val }}$ and $P_{\text {vir }}, \kappa$ and $\left.\kappa_{\text {vir }}\right)$ were refined successively, until convergence.

\section{Results and discussion}

\subsection{Molecular geometry and crystal packing}

The structure at $100 \mathrm{~K}$ is similar to the room-temperature structure (Katrusiak, 1989). Fig. 1 shows the displacementellipsoid representation of the molecule, together with the numbering scheme. The molecule lies in a special position of space group $C 2 / m$, on the mirror plane. The low-temperature analysis gave no evidence for disorder of the methyl group, contrary to the metastable alternative position of the methyl $\mathrm{H}$ atoms found in the room-temperature structure (Katrusiak, 1989).

The bond lengths and angles pattern (given here for the EXP_MUL model) indicates well defined double bonds within the conjugated $\pi$-electron bond system $\mathrm{O}=\mathrm{C}-\mathrm{C}=\mathrm{C}-\mathrm{OH}$. However, the effect of the conjugation is evident: both formally double bonds are longer than typical ones (given in square brackets, International Tables for X-ray Crystallography, 1995): C2-C3 1.3385 (2) A [1.32 A], C1-O1 1.2111 (2) $\AA$ [1.20 ̊] , while the single bonds are significantly shortened: $\mathrm{C} 1-\mathrm{C} 2 \quad 1.4043(7) \AA \quad[1.47 \AA]$ and $\mathrm{C} 3-\mathrm{O} 3$

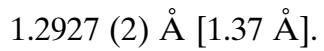

The symmetry coordinates, corresponding to the antisymmetrical in-plane vibration of the $\beta$-diketone fragment, defined as $q_{1}=d_{1}-d_{4}, q_{2}=d_{3}-d_{2}$ can be used as an indicator of the $\pi$-delocalization within this fragment. Because $q_{1}$ and $q_{2}$ are correlated, their sum $q_{\text {sum }}=q_{1}+q_{2}$, a unique antisymmetrical vibration coordinate, can also be regarded as the

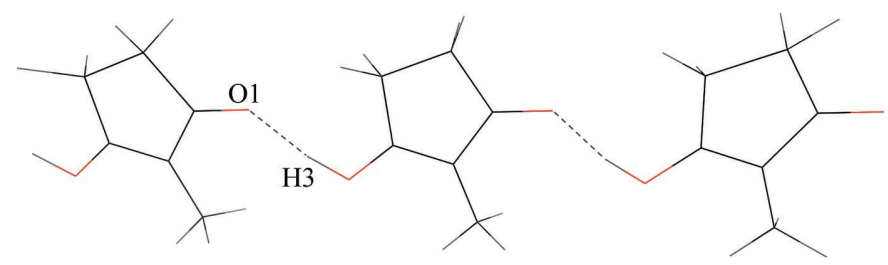

Figure 2

Crystallographic crossed-eyed autostereogram (Katrusiak, 2001) showing the main hydrogen bond motif in a MCPD trimer (translations $\pm \mathbf{c}$ ). The view along the b axis was generated with MoProViewer (Guillot, 2011). 
single indicator of the degree of conjugation (Gilli et al., 1989). The value of $q_{\text {sum }}$ ranges from $+0.320 \AA$ for an ideal, localized enol-keto system to $-0.320 \AA$ for a keto-enol one. For a fully delocalized structure this value should be equal to 0 . In MCPD, the $q_{\text {sum }}$ value is $0.147 \AA$, which indicates a medium degree of delocalization within the $\mathrm{O}=\mathrm{C}-\mathrm{C}=\mathrm{C}-\mathrm{OH}$ bond system.

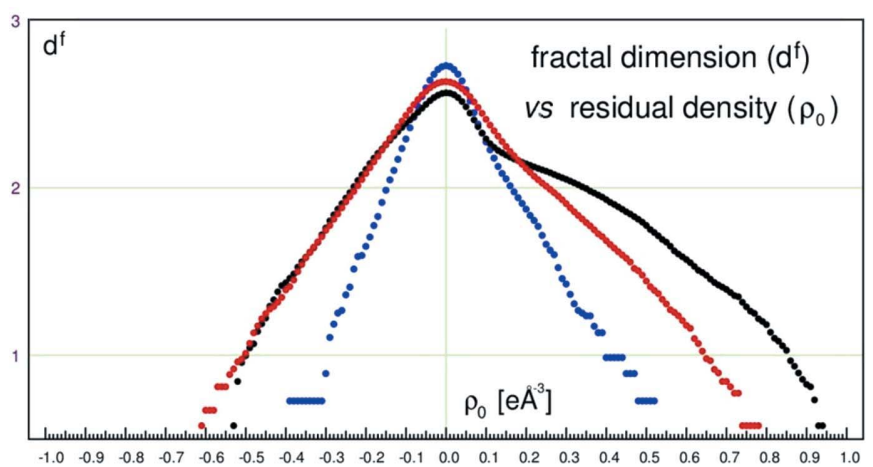

Figure 3

Fractal analysis of the residual electron density. Black: spherical IAM; red: EXP_VIR; blue: EXP_MUL models.

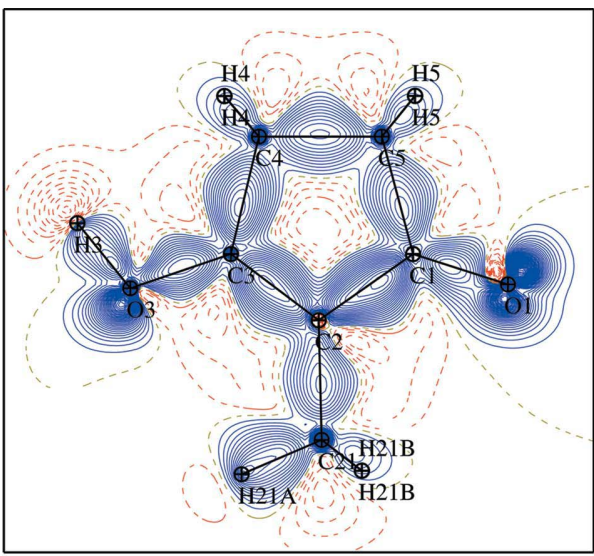

(a)

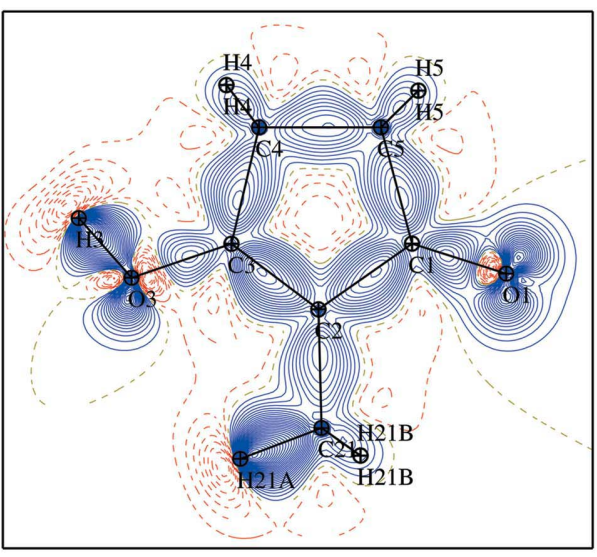

(c)

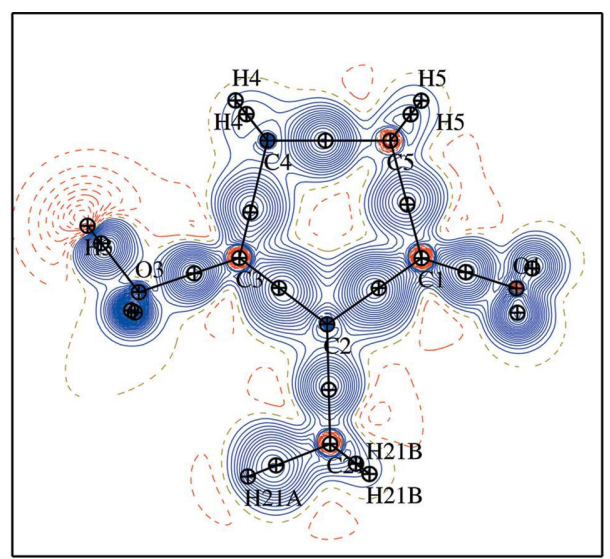

(b)

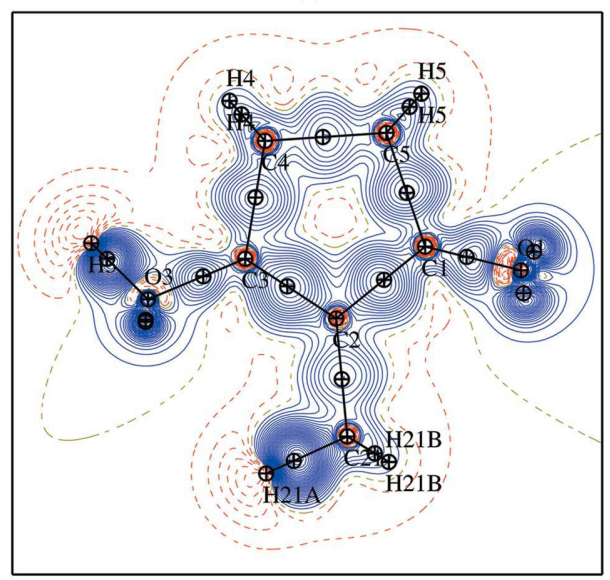

(d)
Figure 4

Static deformation electron-density map in the plane $y=0$ of the molecule. $(a)$ EXP_MUL; $(b)$ EXP_VIR; $(c)$ THEO_MUL; $(d)$ THEO_VIR. Contour level: \pm 0.05 e $\AA^{-3}$. Positive: solid blue lines; negative: dashed red lines; dashed yellow lines. $-x+\frac{1}{2},-y+\frac{1}{2},-z+2$.
Table 3

Geometrical parameters $\left(\AA,{ }^{\circ}\right)$ of the hydrogen bonds in the experimental multipolar atom model.

\begin{tabular}{lllll}
\hline$D-\mathrm{H} \cdots A$ & $D-\mathrm{H}(\AA)$ & $\mathrm{H} \cdots A(\AA)$ & $D \cdots A(\AA)$ & $D-\mathrm{H} \cdots A\left(^{\circ}\right)$ \\
\hline $\mathrm{O} 3-\mathrm{H} 3 \cdots \mathrm{O} 1^{\mathrm{i}}$ & 1.02 & 1.53 & $2.5371(1)$ & 169.0 \\
$\mathrm{C} 4-\mathrm{H} 4 \cdots 1^{\mathrm{i}}$ & 1.09 & 2.67 & $2.9521(2)$ & 93.5 \\
$\mathrm{C} 21-\mathrm{H} 21 A \cdots \mathrm{O} 3^{\mathrm{ii}}$ & 1.06 & 2.74 & $3.5574(2)$ & 134.0 \\
$\mathrm{C} 4-\mathrm{H} 4 \cdots 3^{\text {iii }}$ & 1.09 & 2.79 & $3.3442(1)$ & 111.3 \\
$\mathrm{C} 21-\mathrm{H} 21 B \cdots{ }^{\text {iv }}$ & 1.06 & 2.75 & $3.5214(1)$ & 130.1 \\
\hline
\end{tabular}

Symmetry codes: (i) $x, y, z-1$; (ii) $-x,-y,-z+1$; (iii) $-x+\frac{1}{2},-y+\frac{1}{2},-z+1$; (iv)

The crystal packing is mainly determined by a strong intermolecular $\mathrm{O}-\mathrm{H} \cdots \mathrm{O}$ hydrogen bond between molecules connected by unit-cell translations along [001] (Fig. 2). The hydrogen-bond configuration is anti/SYN (in 1,3-cyclopentanedione it is anti/ANTI), and the $\mathrm{O} \cdots \mathrm{O}$ distance, 2.5371 (1) $\AA$, lies well within the typical range for $\beta$-diketoalkanes. Four weak $\mathrm{C}-\mathrm{H} \cdots \mathrm{O}$ contacts (Table 3 ) also contribute to crystal stability.

\subsection{Residual electron density}

The refinement statistics listed in Table 2 give a general idea of the quality of the models. The multipolar (EXP and THEO) models show $10 \%$ lower $R$ factors compared with their counterpart using real and virtual spherical charges. This can be explained by the larger flexibility of the multipolar atom model for which the number of parameters describing the charge density is larger compared with the virtual atom model (Table 2). A similar tendency in $R$ factors was observed in an earlier study (Dadda et al., 2012).

The quality of the charge-density models was also assessed by analyzing the Fourier residual electron density maps. The residual maps are shown in Fig. S1 in the plane $y=0$ of MCPD. In all the residual maps, most of the bonding and lone pair electron density is modelled. All peaks observed on the residual map are low except for a few peaks around the $\mathrm{H}$ atoms and the Lp sites. The r.m.s. of the residual electron density is also indicated in Table 2. The r.m.s. value is $18 \%$ lower for the 
EXP_MUL model compared with EXP_VIR; for the theoretical models, the same reduction is observed.

Generally, an accurate electron density modeling should result in a fit of the diffraction data good enough to ensure that only random noise appears in the residual maps. The normal probability plots of the residual density (Zhurov et al., 2008) are given for the EXP_MUL, EXP_VIR and EXP_IAM models in Fig. S2, while the weighting scheme was adjusted to have a goodness-of-fit equal to unity in each model. The plots are globally similar for the MUL and VIR models and are very close to normal probability except for the largest residual density values. The EXP_MUL plot shows a better adjustment to normal probability for the large residual peaks compared with EXP_VIR, notably the positive ones. On the other hand, the IAM model is clearly far from normal probability, indicating the over-representation of large positive residual density values.

A fractal analysis of the residual density is shown in Fig. 3 for the three models (Meindl \& Henn, 2008). The EXP_IAM refinement yields a non-symmetric plot with a shoulder appearing on the positive part of $\Delta \rho$, indicating excess positive residual electron density, due to the inadequacy of the spherical atom to model the bonding density features. The EXP_MUL $\Delta \rho$ map shows a nearly symmetric fractal dimension distribution plot indicating a good quality of the diffraction data and model. The EXP_VIR fractal plot is more symmetric than the EXP_IAM one. On the positive part of $\Delta \rho$, the EXP_VIR plot is intermediary between the multipolar and spherical models. On the negative side, the EXP_VIR plot is not improved compared with the EXP_IAM one. This can be attributed to the fact that the VIR description, unlike the multipolar atoms, cannot model electrondensity depletion features, except spherically distributed electron depletions on atoms.

\subsection{Deformation electron density}

The deformation electron density is defined as the difference between the total molecular density described by the multipolar-atom or virtual and real spherical atom model and the superposition of spherical independent atoms (IAM). The static deformation electron density maps in the plane of the molecule calculated from the four electron density modeling are presented in Fig. 4.

The features of the static deformation electron density map (Fig. 4) are generally consistent with the alternate single-/ double-bond character of the $\mathrm{O}=\mathrm{C} 1-\mathrm{C} 2=\mathrm{C} 3-\mathrm{OH}$ fragment. The heights of the maxima of the EXP_MUL deformation density within this fragment are $c a 0.55,0.95,0.80$ and $0.60 \mathrm{e}^{-3}$, for $\mathrm{O}=\mathrm{C}, \mathrm{C}-\mathrm{C}, \mathrm{C}=\mathrm{C}$ and $\mathrm{C}-\mathrm{OH}$ bonds, respectively (Table S3). Despite the formally single bond character of $\mathrm{C} 1-\mathrm{C} 2$, its bonding electron-density maximum is higher than that of the $\mathrm{C} 2=\mathrm{C} 3$ double bond, presumably due to the resonance with the nearby $\mathrm{C}=\mathrm{O}$ group. This is also observed for the EXP_VIR model. However, in the two theoretical models, the reverse is observed and the bond peak heights are, this time, in accordance with the formal bonding order of $\mathrm{C} 1-\mathrm{C} 2$ and $\mathrm{C} 2=\mathrm{C} 3$.

It should be noted for the EXP_MUL model that the 0.70 e $\AA^{-3}$ peak on the formally single $\mathrm{C} 3-\mathrm{C} 4$ bond is higher than the peaks related to the three other single $\mathrm{C}-\mathrm{C}$ bonds $(\mathrm{C} 4-\mathrm{C} 5, \mathrm{C} 5-\mathrm{C} 1, \mathrm{C} 2-\mathrm{C} 21)$, not involved in the resonance environment, for which the maxima are all lower than $0.55 \mathrm{e}^{-3}$. This trend is not necessarily observed for the three other models. The THEO_VIR model shows the lowest $\mathrm{C}-\mathrm{C}$ bonding electron density for the $\mathrm{C}-\mathrm{CH}_{3}$ and the $\mathrm{CH}_{2}-\mathrm{CH}_{2}$

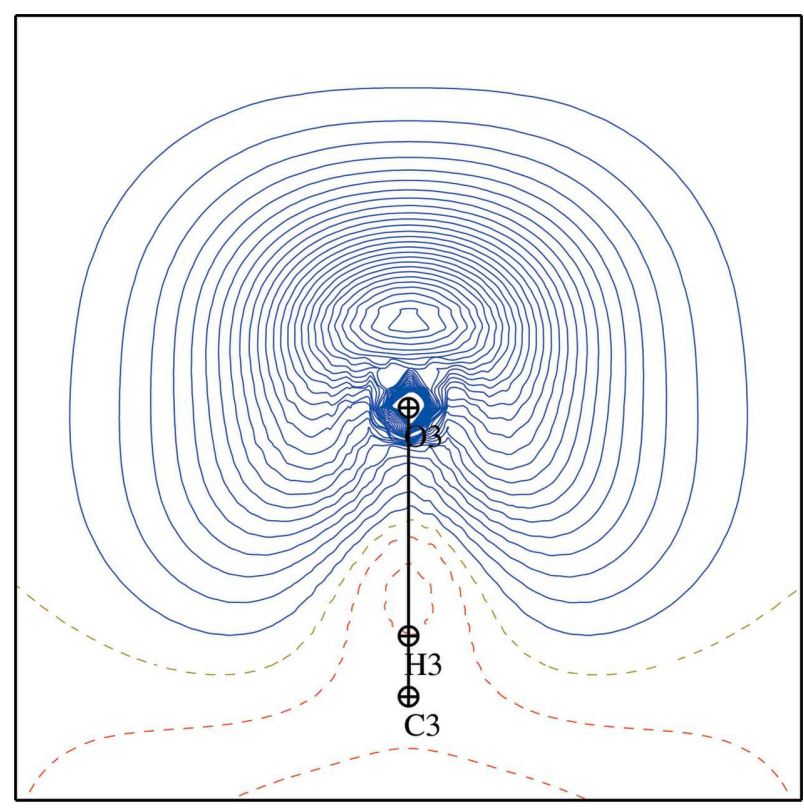

(a)

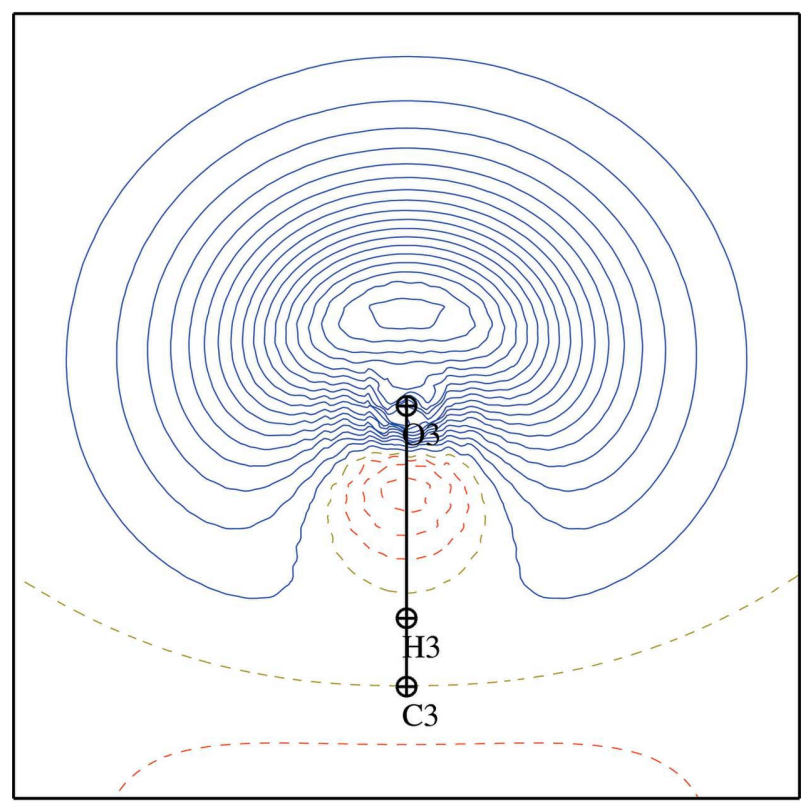

(b)

Figure 5

Static deformation map of the multipolar electron density in the plane bisecting $\mathrm{C} 3-\mathrm{O} 3-\mathrm{H} 3$ showing the merged lobes of the lone pairs: $(a)$ Experimental; (b) theoretical. Contours as in Fig. 3. 
moieties, which are formally single bonds and far from resonant groups.

The static deformation map (Figs. 4 and 5) also shows the electron lone pair lobes ( $\mathrm{Lp}$ ) of the two $\mathrm{O}$ atoms. The lone pairs of the carbonyl O1 atom lie - by crystallographic symmetry - in the mirror plane of the molecule. The Lp$\mathrm{O} 1-\mathrm{C} 1$ angles (where $\mathrm{Lp}$ is defined as the peak of a lone pair lobe deformation density) are, as expected, close to $120^{\circ}$. These results are consistent with other charge-density determinations for carbonyl fragments [e.g. in glycyl-L-threonine dihydrate; Benabicha et al., 2000; and in DL-alanyl-methionine (Guillot, Muzet et al., 2001)]. The two carbonyl electron lone

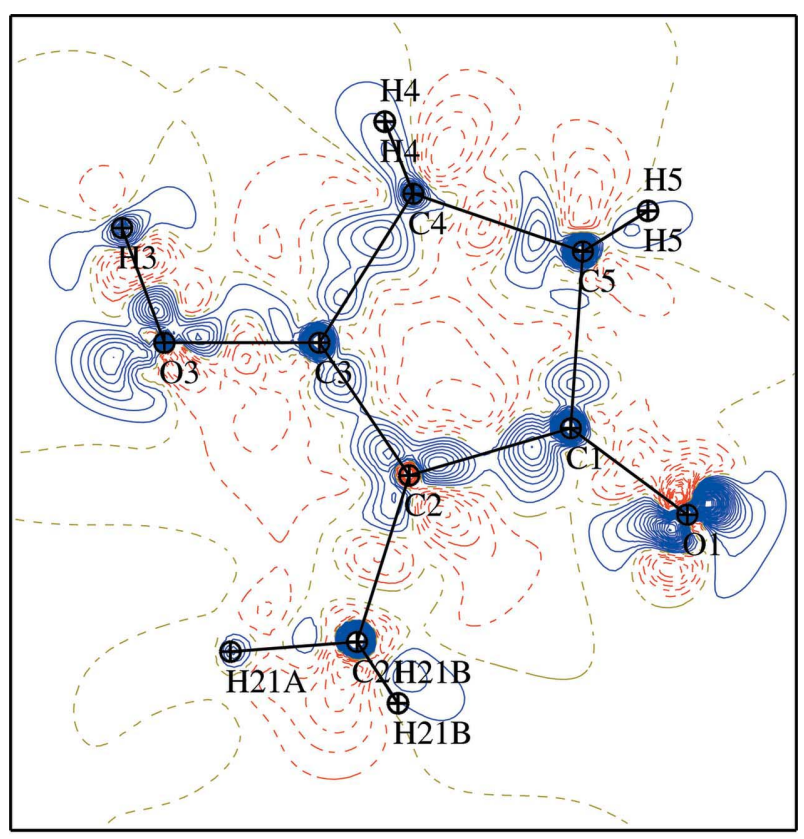

(a)

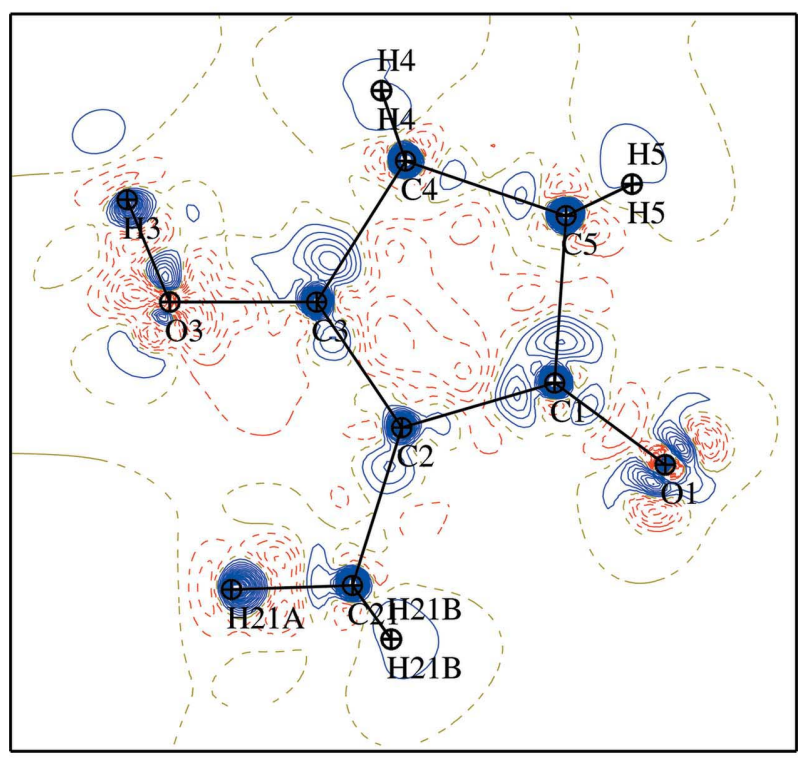

(b)

Figure 6

Difference between models of static deformation electron density in the plane $y=0$ of the molecule. (a) EXP_MUL-EXP_VIR; (b) THEO_MUL-THEO_VIR. Contours as in Fig. 4. pairs are slightly dissymmetric in the experimental models, but not in the theoretical ones.

Fig. 5 shows the deformation electron density in the plane bisecting the $\mathrm{COH}$ group, where the two electron lone pairs of the hydroxyl $\mathrm{O}$ atom are localized. Only one deformation electron density maximum is observed in that plane. This merging of the two electron lone pairs is due to conjugation with the five-membered ring which gives a partial $s p^{2}$ character to the $\mathrm{O} 3$ atom. A similar effect was observed for the $\mathrm{C}-\mathrm{O}-\mathrm{C}$ ester $\mathrm{O}$ atom of coumarin 214 (Munshi et al., 2010) located within an aromatic cycle, both from experimental and theoretical data. The other $\mathrm{C}-\mathrm{O}-\mathrm{C}$ oxygen atom of the ethyl ester side chain shows, contrarily, two distinct lone-pair lobes.

The different lone-pair configurations observed in alcohols, phenols, esters and ethers was described and discussed in Ahmed et al. (2013). The lone pairs in deformation density maps issued from theoretical structure factors also appear to be slightly closer in phenols than in alcohols, due to conjugation with the aromatic ring. In some experimental chargedensity studies at $100 \mathrm{~K}$, phenols show merged lone pair lobes in leu-enkephaline (Pichon-Pesme et al., 1992) or $N$-acetyl-Ltyrosine ethyl ester (Dahaoui et al., 1999). However, in more recent studies carried out at ultra low temperature $20 \mathrm{~K}$, two distinct lone pairs are visible in the electron density: estrone (Zhurova et al., 2006), 17 $\beta$-estradiol-urea (Parrish et al., 2006), diethylstilbestrol and dienestrol (Yearley et al., 2008).

The C3-O3 bond length 1.2927 (1) $\AA$ is significantly shorter than the phenol $\mathrm{C}-\mathrm{O}$ bond length, which is, for example, $1.365 \AA$, in the estrone structure at $20 \mathrm{~K}$; this is another confirmation of a certain degree of conjugation within the MCPD molecule.

The difference between the deformation electron-density maps (Fig. 6) indicates the dissimilarities between the models. The largest discrepancy is found around the carbonyl O1 atom and hydroxyl O3 atom between EXP_MUL and EXP_VIR models. This is to be related to the different modelling of the Lp electron density on the $\mathrm{O}$ atoms in the MUL and VIR models. The theoretical map comparison shows a systematic larger electron density around the atomic nuclei in THEO_MUL than in THEO_VIR, as in the latter model, the valence populations $P_{\text {val }}$ of the real atoms are depleted in favour of the $P_{\text {vir }}$ parameters. This is also observed in most of the cases in the experiment models.

The difference between the B3LYP electron density obtained directly from CRYSTAL09 (Dovesi et al., 2010) and the THEO_MUL/THEO_VIR models is shown in Fig. S6. The THEO_MUL model shows generally a nice match with the B3LYP electron density, except on the $\mathrm{H}$ atoms and $\mathrm{H}-X$ bonds. The THEO_VIR model shows discrepancies in the same regions, but also on and around the atom nuclei.

In order to compare electron densities, the r.m.s. values and the correlation coefficients between the deformation electron densities obtained from the four models were computed (Table 4). The THEO_MUL and THEO_VIR maps agree qualitatively well and display the highest correlation coefficient $\sigma=0.95$. The correlation between the EXP_MUL and the EXP_VIR models is lower, presumably because the 
structural and thermal parameters are refined in addition to the charge density itself. The lowest correlation is found between EXP_MUL and THEO_VIR models.

The r.m.s. magnitude of the deformation electron density is shown in Table 4 for the four models. For example, the r.m.s.

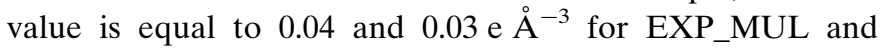
EXP_VIR, respectively.

For all four models, the deformation electron-density peaks are centered on the $\mathrm{C}-\mathrm{C}, \mathrm{C}=\mathrm{C}$, and $\mathrm{C}-\mathrm{OH}$ bonds. For instance, the $\mathrm{C} 1-\mathrm{C} 2$ bond of the MCPD ring shows deformation peak heights ranging from 0.95, 0.85, 0.65 and 0.70 e $\AA^{-3}$ for EXP_MUL, EXP_VIR, THEO_MUL and THEO_VIR, respectively (Table S3). The bonding electron density peaks are generally of similar height in the multipolar and VIR models, but globally r.m.s. $\left(\rho_{\text {def }}\right)$ is $20-25 \%$ smaller for the VIR models. The Lp lobes of the $\mathrm{C}=\mathrm{O}$ group are strongest in the EXP_MUL and THEO_VIR maps.

The experimental deformation densities also show smaller r.m.s. values than the theoretical ones. The EXP_VIR and THEO_VIR maps show systematically high negative defor-

\section{Table 4}

Correlation coefficient between the deformation electron densities $\rho_{\text {def }}$ calculated from the four charge-density models.

The density B3LYP resulting directly from the software CRYSTAL is also compared. The r.m.s. value of $\rho_{\text {def }}$ is given in italics on the diagonal of the table.

\begin{tabular}{llllll}
\hline Model & EXP_MUL & EXP_VIR & THEO_MUL & THEO_VIR & B3LYP \\
\hline EXP_MUL & 0.040 & 0.90 & 0.87 & 0.83 & 0.86 \\
EXP_VIR & - & 0.030 & 0.85 & 0.90 & 0.87 \\
THEO_MUL & - & - & 0.050 & 0.95 & 0.94 \\
THEO_VIR & - & - & - & 0.045 & 0.91 \\
B3LYP & - & - & - & - & 0.057 \\
\hline
\end{tabular}

mation electron-density peaks in the close vicinity of the atomic nuclei. When the original deformation electron density B3LYP obtained directly from quantum calculation is considered, the THEO_MUL model has a slightly higher correlation coefficient $\sigma=0.94$ than the THEO_VIR model, which is in accordance with its larger number of adjustable parameters. In conclusion, the experimental and theoretical charge-density models are quite satisfactory in representing the deformation densities.

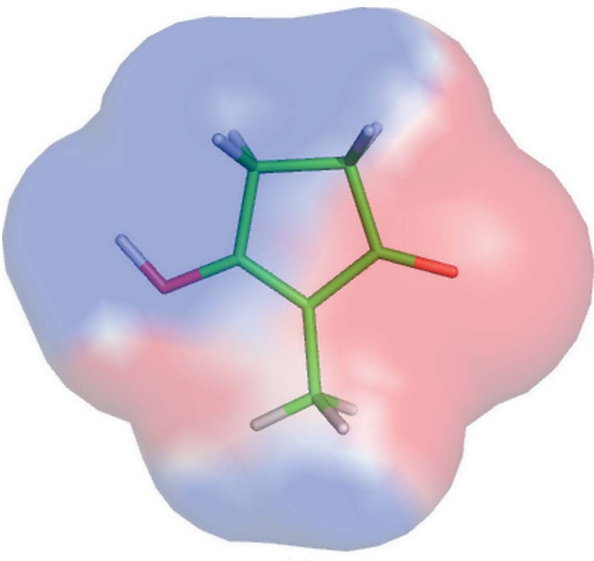

(a)

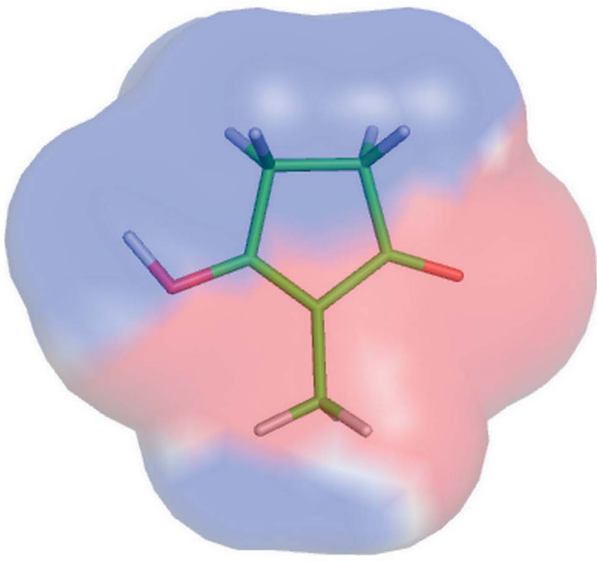

(c)

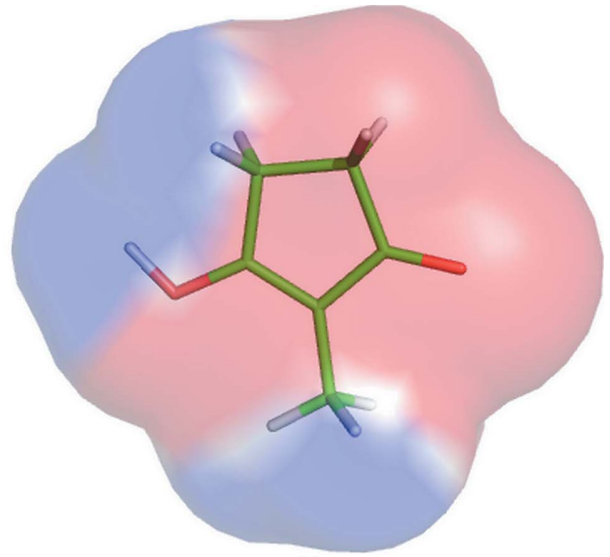

(b)

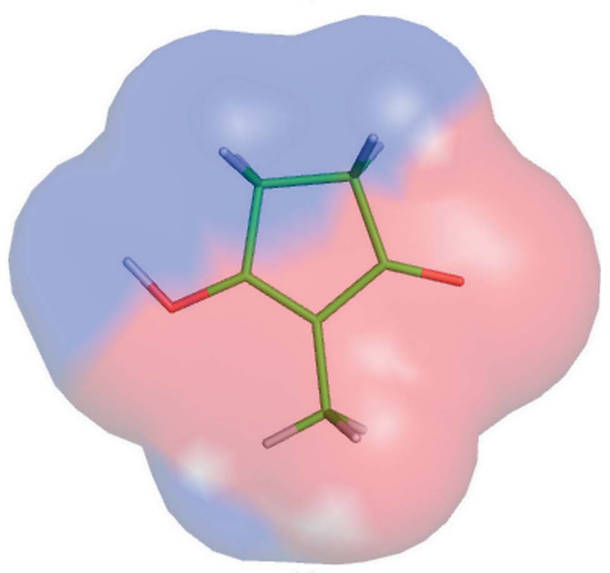

(d)
Figure 7

Electrostatic potential mapped on the van der Waals surface of the MCPD molecule on the 0.01 e $\AA^{-3}$ isocontour surface. The maximum negative (red) and positive (blue) values of the ESP correspond to the values -0.05 and 0.05 e $\AA^{-1}$, respectively. (a) EXP_MUL, $(b)$ EXP_VIR, $(c)$ THEO_MUL and (d) THEO_VIR models.

\subsection{Electrostatic potential}

The electrostatic potential (ESP) is an important property that can be derived from electron density distribution. The electrostatic properties are generated using the VMoPro software on an isolated molecule extracted from the crystal lattice. This property is mainly used to investigate potential molecular interactions. The electrostatic potential generated at the $0.001 \mathrm{e} \mathrm{Bohr}^{-3}$ electron density surface is coloured according to the ESP value in Fig. 7.

For the four charge-density models, the maps show a negative potential area around the carbonyl $\mathrm{O} 1$ atom and a smaller one close to the hydroxyl $\mathrm{O} 3$ atom (Fig. 7). The electrostatic potential around the O3 hydroxyl atom is, as expected, less negative than around the carbonyl group, thus confirming the stronger hydrogen-bond accepting ability of the carbonyl group in comparison with the hydroxyl one. The weak $\mathrm{C} 21-\mathrm{H} 21 A \cdots \mathrm{O} 3$ $(-x,-y,-z+1)$ hydrogen-bond interaction takes place in the region of a negative potential close to the $\mathrm{O} 3$ atom (Fig. 7). The strong 
Table 5

Correlation coefficients between the electrostatic potentials $V$ calculated from the four electron-density models applied on the experimental crystal structure.

The statistics on $V\left(\mathrm{e} \AA^{-1}\right)$ are computed in a volume from 0 to $2 \AA$ distance outside the van der Waals surface of MCPD. The r.m.s. values are given in italics diagonally across the table

\begin{tabular}{lllll}
\hline Model & EXP_MUL & EXP_VIR & THEO_MUL & THEO_VIR \\
\hline EXP_MUL & 0.027 & 0.93 & 0.90 & 0.92 \\
EXP_VIR & - & 0.027 & 0.75 & 0.80 \\
THEO_MUL & - & - & 0.035 & 0.99 \\
THEO_VIR & - & - & - & 0.039
\end{tabular}

Table 6

Electrostatic interaction energy $\left(\mathrm{kJ} \mathrm{mol}^{-1}\right)$ between dimers in contact in the crystal packing, computed from different models.

\begin{tabular}{|c|c|c|c|c|c|}
\hline Data model & EXP MUL & EXP VIR & THEO MUL & THEO VIR & Contact \\
\hline$x, y, z-1$ & -97.72 & -74.12 & -133.70 & -118.92 & $\mathrm{H} 3 \cdots \mathrm{O} 1$ \\
\hline$-x+\frac{1}{2},-y-\frac{1}{2},-z+1$ & -31.89 & -14.41 & -36.20 & -39.70 & $\mathrm{C} 21 \cdots \mathrm{H} 4$ \\
\hline$-x+1, y,-z+2$ & -7.95 & 2.45 & -17.74 & -22.42 & $\mathrm{H} 5 \cdots \mathrm{H} 5$ \\
\hline$-x, y,-z+1$ & -6.08 & -5.13 & -9.18 & -7.01 & $\mathrm{H} 21 A \cdots \mathrm{H} 21 A$ \\
\hline$x-\frac{1}{2}, y-\frac{1}{2}, z$ & 1.26 & -0.27 & 2.83 & 4.67 & $\mathrm{H} 5 \cdots \mathrm{H} 21 B$ \\
\hline$-x, y,-z+2$ & 0.29 & -1.18 & -2.97 & -2.87 & $\mathrm{H} 21 B \cdots \mathrm{H} 21 B$ \\
\hline$-x+\frac{1}{2}, y-\frac{1}{2},-z+2$ & -5.92 & -4.91 & 0.71 & 2.70 & $\mathrm{O} 1 \cdots \mathrm{H} 21 B$ \\
\hline$x-\frac{1}{2},-y+\frac{1}{2}, z$ & 1.26 & -0.27 & 2.83 & 4.67 & $\mathrm{H} 21 B \cdots \mathrm{H} 5$ \\
\hline$-x+1, y,-z+1$ & -7.44 & -8.79 & 1.32 & 4.04 & $\mathrm{H} 4 \cdots \mathrm{H} 5$ \\
\hline R.m.s. value & 34.0 & 26.0 & 46.7 & 42.6 & - \\
\hline
\end{tabular}

Table 7

Correlation coefficient between the electrostatic interaction energies computed for the four electron density models of MCPD.

\begin{tabular}{lllll}
\hline Model & EXP_MUL & EXP_VIR & THEO_MUL & THEO_VIR \\
\hline EXP_MUL & - & 0.98 & 0.99 & 0.98 \\
EXP_VIR & - & - & 0.96 & 0.97 \\
THEO_MUL & - & - & - & 0.99 \\
\hline
\end{tabular}

$\mathrm{O} 1 \cdots \mathrm{H} 3$ hydrogen bond clearly shows a valley of positive potential along the bond (Fig. S4) path, as discussed earlier by Espinosa et al. (1996) and by Klooster et al. (1992).

In all four models, the electro-positive and electro-negative potentials are well separated. The negative potential around the carbonyl $\mathrm{O}$ atom is strongest in the EXP_VIR model compared with the two multipolar models (EXP_MUL and THEO_MUL). To compare quantitatively the models, the ESP was computed in a volume around the van der Waals surface of the MCPD molecule, at a distance ranging from 0 to $2 \AA$ (Table 5). Good quantitative agreement is observed between the ESP r.m.s. values for the four models: the THEO_VIR model shows a slightly higher ESP r.m.s. value compared with EXP_VIR. Globally, the theoretically derived ESPs show larger r.m.s. magnitudes than the experimental ones.

The correlation coefficients between the electrostatic potential issued from the four different charge-density models are also listed in Table 5. The best correlation is observed in the present study between THEO_MUL and THEO_VIR (99\%), followed by EXP_MUL and EXP_VIR (93\%). The two models refined versus the theoretical structure factors display a very high correlation, as already observed for the deformation electron density (Table 4).

\subsection{Electrostatic interaction energy}

The software VMoPro allows the electrostatic energy $\left(E_{\mathrm{es}}\right)$ of molecular dimers within the Buckingham-type approximation to be accurately calculating. This property is an invaluable tool for understanding molecular interactions. The $E_{\mathrm{es}}$ values were computed for all the pairs of interacting molecules occurring in the crystal packing (Table 6) for the four different electron-density models. To compare the models quantitatively, the correlation coefficients and r.m.s. values are presented in Table 7. In all cases, the strong hydrogen bond $\mathrm{H} 3 \cdots \mathrm{O} 1$ has the strongest $E_{\mathrm{es}}$ value and accounts for most of the electrostatic energy. The different $E_{\text {es }}$ values show some variability with the electron density model used, as already observed by Bạk et al. (2011).

The EXP_VIR model reproduces the electrostatic interaction energies obtained from the EXP_MUL model with a $\sigma=98 \%$ correlation. Comparison of the EXP_VIR energies with those calculated with THEO_MUL and THEO_VIR models shows a low attenuation in $E_{\text {es }}$. The r.m.s. values are equal to 34.0, 26.0, 46.7 and $42.6 \mathrm{~kJ} \mathrm{~mol}^{-1}$ for EXP_MUL, EXP_VIR, THEO_MUL and THEO_VIR, respectively. The THEO_MUL $E_{\text {es }}$ exhibits the largest r.m.s. value compared with the other models. Concerning the experimental models, the magnitude is on average $24 \%$ lower for the EXP_VIR model compared with EXP_MUL.

Good quantitative accordance is observed between the two theoretical models with similar r.m.s. $\left(E_{\mathrm{es}}\right)$ values and a high correlation coefficient $\sigma=0.99$.

Strong correlations are observed between the EXP_MUL and EXP_VIR models $(\sigma=0.98)$ and between the EXP_MUL and THEO_MUL models $(\sigma=0.99)$. It is concluded that the new models EXP_VIR and THEO_VIR that have been used in this study are demonstrated to be reliable for computing electrostatic potential and intermolecular interaction energy and can therefore be utilized for protein interaction modeling.

\subsection{Dipole moments}

To judge the quality of dipole obtained from the calculation based on the multipolar and virtual atom model refined with experimental structure factors, we completed our analysis to include quantum mechanical methods: (i) density functional theory in its B3LYP/6-31G(d,p), (ii) second-order MøllerPlesset (MP2) (Møeller \& Plesset, 1934). The dipole moment in vacuo was obtained by ab initio calculations $[6-31 \mathrm{G}(\mathrm{d}, \mathrm{p})$ and MP2] computed directly from the wavefunctions; these calculations were performed with GAUSSIAN09 program package (Frisch et al., 2009). 
Table 8

Module of the molecular dipole moment $\mu$ (Debye) of MCPD for the different models.

\begin{tabular}{llllll}
\hline Method & EXP_MUL & EXP_VIR & THEO_MUL & THEO_VIR & $\begin{array}{l}\text { Isolated molecular } \\
\text { B3LYP/MP2 }\end{array}$ \\
\hline$\mu$ & 4.90 & 5.00 & 6.34 & 7.45 & $4.92 / 5.55$ \\
\hline
\end{tabular}

Katrusiak (1991) on the basis of simple MNDO calculations. This result supports the crucial role of the distribution of dipole moments in the stability of the MCPD crystal in comparison with the other simple cyclic $\beta$-diketoalkanes.

\subsection{Topological analysis of the covalent bonds}

More fundamental insight into the electronic characterization of bonds can be obtained by means

The modules of the dipole moments, calculated from different approaches, are listed in Table 8. The dipole moment of MCPD has been estimated to be 4.90 Debye using the EXP_MUL model and 5.00 Debye using the EXP_VIR model. The EXP_MUL dipole moment $|\mu|$ is very close to that of the EXP_VIR model. The $|\mu|$ values from the MP2 and B3LYP methods agree best with EXP_MUL and EXP_VIR models. The dipole moments derived from THEO_MUL and THEO_VIR approaches are higher than those computed from the experimental data refinements, as was already observed for the electrostatic potential.

Fig. 8 shows the directions of the dipole moment vectors, the origin being at the MCPD centre of mass. For all models, the orientation of the dipole moment is dominated by the charges of the carbonyl and hydroxyl groups. The EXP_MUL and EXP_VIR dipole moments have similar directions as the angle between the two vectors is $0.8^{\circ}$. The THEO_MUL and THEO_VIR dipole moment vectors deviate from the EXP_MUL one by 5.1 and $8.0^{\circ}$, respectively. The dipole vectors computed from the two theoretical models have practically the similar direction. These results are consistent with our recent study on dipole moments calculated with different electron density models in 2-methyl-4-nitro-1phenyl-1H-imidazole-5-carbonitrile (Poulain-Paul et al., 2012).

The EXP_MUL dipole moment (4.90 Debye) calculated for the molecule in the crystal has nearly the same direction as the c cell axis, which is in agreement with that postulated by

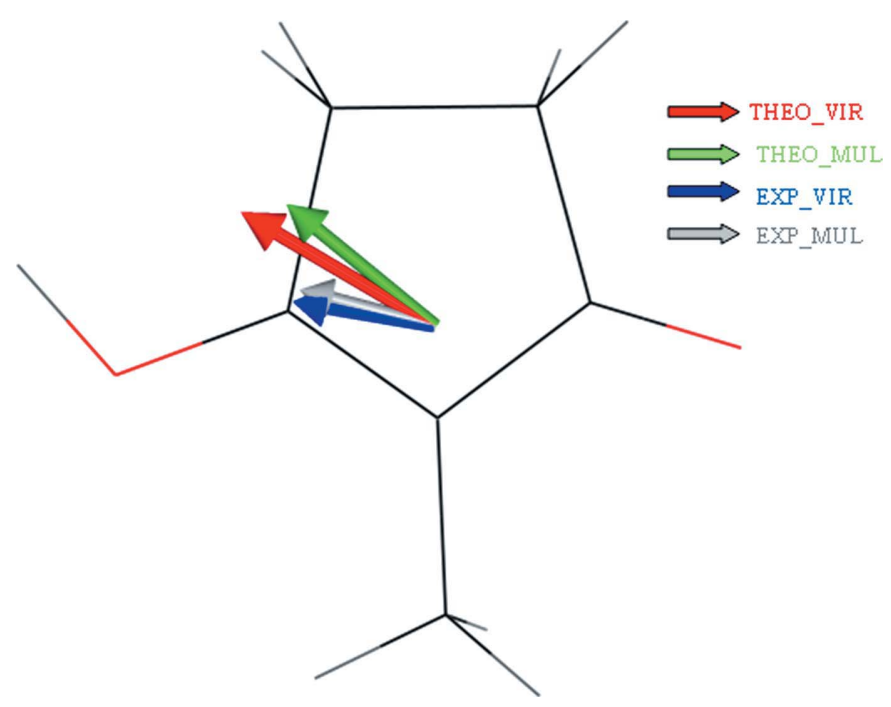

Figure 8

Dipole moment of the MCPD compound represented for the four chargedensity models. The origin is at the centre of mass. of topological analysis of charge-density distribution. According to the atoms-in-molecules (AIM) theory (Bader, 1990) 'the topology of the charge density $\rho$ yields a faithful mapping of the chemical concepts of atoms, bonds and structure'. The multipolar models yield a critical point on all bonds of MCPD and a basin around each atom.

For the VIR models, each non-H atom of MCPD shows a well defined atomic basin (Fig. 9). $\mathrm{H}-\mathrm{C}$ hydrogen atoms display a very small basin around the nucleus. On the hydroxyl group, the $\mathrm{H} 3$ atom is included in the basin of the carrier $\mathrm{O}$ atom (Fig. 9). $\mathrm{H}$ atoms have no core electrons and in the EXP_VIR model, the $\mathrm{H} 3$ atom has a particularly low $P_{\text {val }}=$ 0.28 e value, which explains the absence of an atomic basin. The map shown in Fig. 9 is static for all atoms, as thermal deconvolution was achieved for all real and virtual atoms.

Therefore, the topological analysis of covalent bonds is performed only on the EXP_MUL model.

Table 9 lists details of the bond-critical points and Fig. 10 shows the Laplacian map in the plane of the molecule and of the $\mathrm{O} 3-\mathrm{H} 3 \ldots \mathrm{O} 1$ hydrogen bond. These data confirm the above-mentioned features: the alternate double/single bond character within the conjugated fragment. The Laplacian values suggest a larger degree of charge concentration at

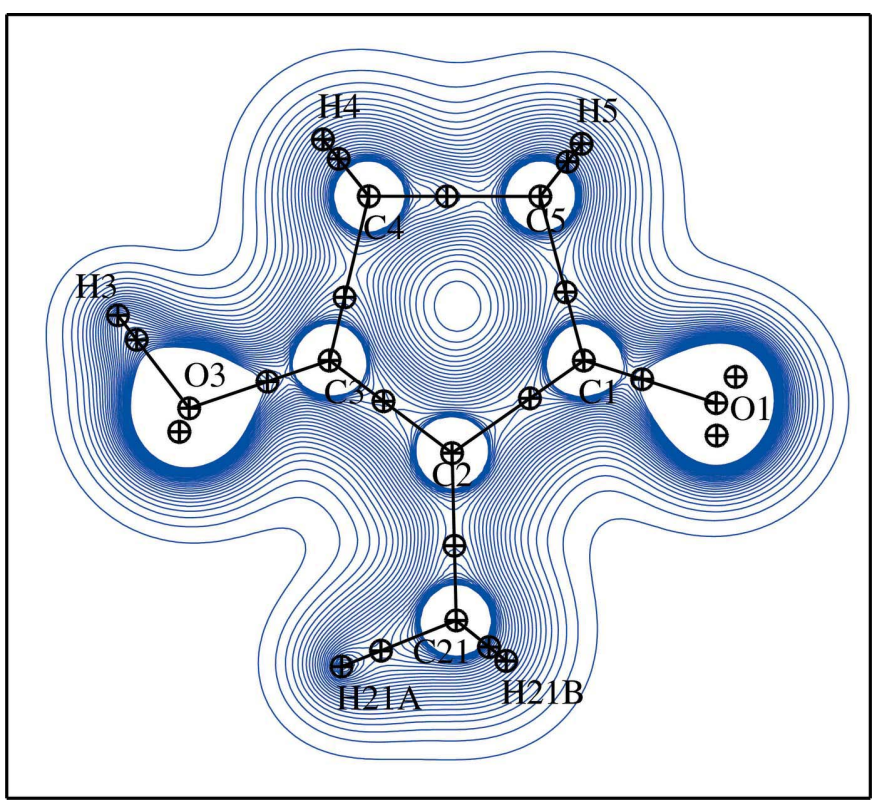

Figure 9

Total static electron density in the plane $y=0$ of the MCPD for the

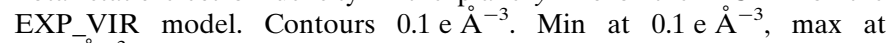
$3.0 \mathrm{e} \AA^{-3}$. 
Table 9

Topological analysis of the EXP_MUL total electron density $\rho$ at the critical points of the covalent bonds and of the $\mathrm{O} \cdots \mathrm{H}-\mathrm{O}$ hydrogen bond.

$d_{12}$ is the interatomic distance, $d_{1 \mathrm{cp}}$ and $d_{2 \mathrm{cp}}(\AA)$ are the distance between the first/second atom and the CP; $\rho\left(r_{\mathrm{cp}}\right)$ is the electron density (e $\left.\AA^{-3}\right)$; $\nabla^{2} \rho\left(r_{\mathrm{cp}}\right)$ is the Laplacian $\left(\mathrm{e} \AA^{-5}\right) ; \lambda_{1}, \lambda_{2}, \lambda_{3}$ are the eigenvalues of the Hessian matrix $\left(\mathrm{e} \AA^{-5}\right) ; \epsilon$ the ellipticity.

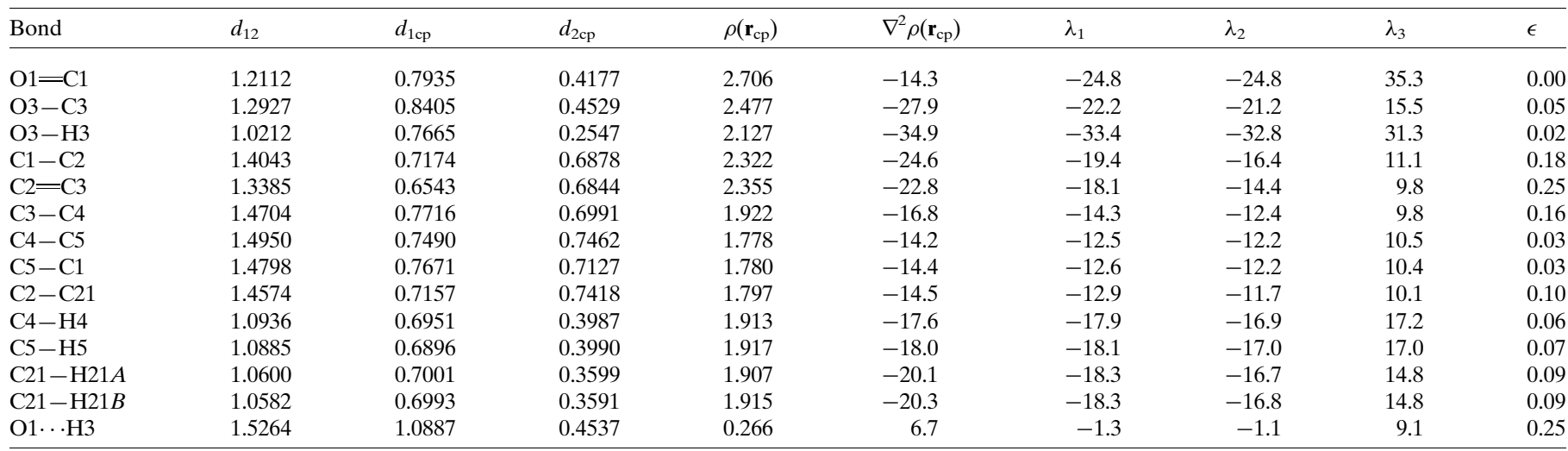

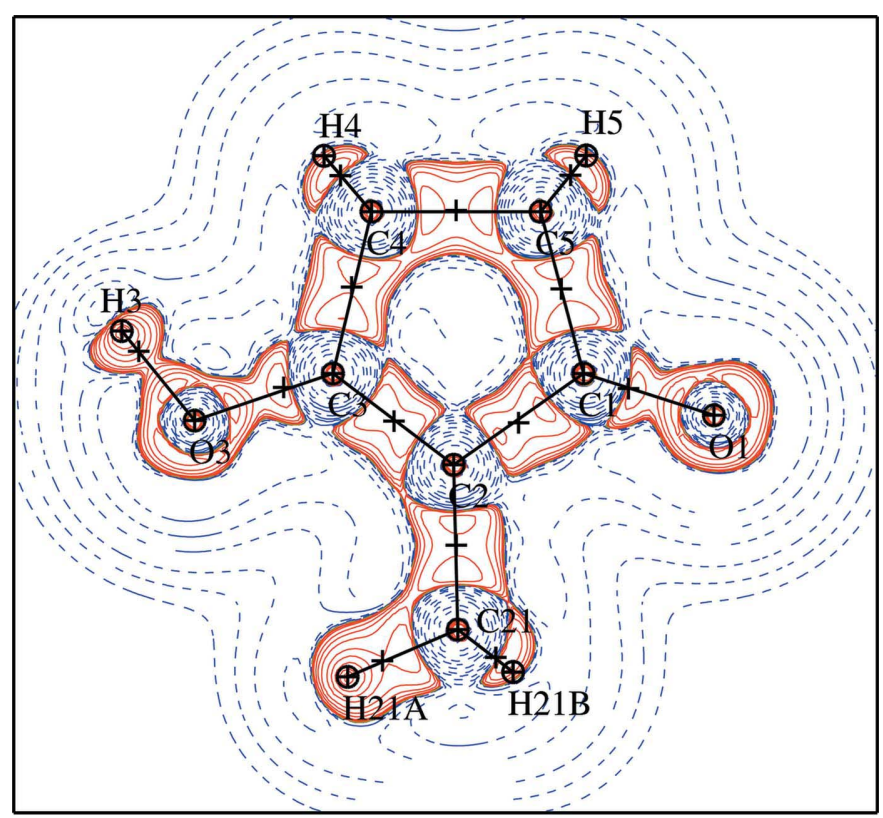

(a)

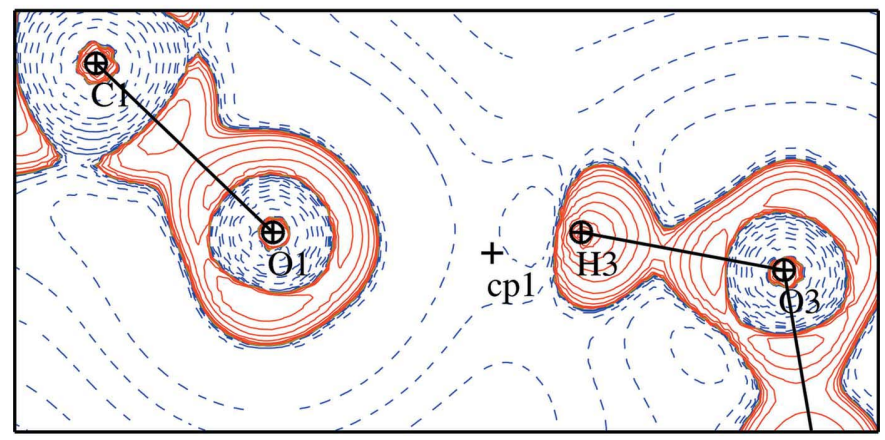

Figure 10

(b)

Laplacian of the EXP_MUL electron density $(a)$ in the plane $y=0$ of the molecule and $(b)$ in the $\mathrm{O} 1 \cdots \mathrm{H} 3-\mathrm{O} 3$ plane of the strong hydrogen bond. Contour level: $\pm 2,4,8 \times 10^{n}$ e $\AA^{-5}, n=-1,0,1$. Positive: solid lines; negative: dashed lines. Bond CPs are also shown. conjugated bond CPs (values between -16.8 and -24.7 e $\AA^{-5}$ ) compared with single $\mathrm{C}-\mathrm{C}$ bonds $(-14.6$ to

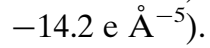

The ellipticities of $\mathrm{C}-\mathrm{C}$ bonds within the conjugated fragment are consistent with the resonance model. For the $\mathrm{C} 2-\mathrm{C} 3$ bond, the value $\epsilon=0.25$ (as well as the values $\rho=$ 2.35 e $\AA^{-3}$ and $\nabla^{2} \rho=-22.8$ e $\AA^{-5}$ at the critical point) are close to the values found in benzene rings. For example, in benzoylacetone (Madsen et al., 1998) values of $\epsilon$ are in the

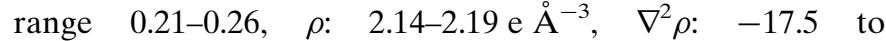
-18.3 e $\AA^{-5}$; in 1-phenyl-4-nitroimidazole (Kubicki et al., 2002) the respective ranges are $0.17-0.22 ; 2.02-2.09 ;-17.3$ to $-18.9)$. The ellipticity value $\epsilon=0.18$ for the formally single $\mathrm{C} 1-\mathrm{C} 2$ bond, which is nearby the $\mathrm{C} 1=\mathrm{O} 1$ group, is higher than for the other formally single bonds in the structure; the same is true for the $\rho$ and $\nabla^{2} \rho$ values.

The small ellipticity values for the $\mathrm{C}-\mathrm{O}$ and $\mathrm{C}=\mathrm{O}$ bonds can be explained by the observation of Cheeseman et al. (1988) that for heteroatomic bonds with a large charge transfer, the ellipticity at the bond critical point is not a sensitive indicator of the $\pi$-contribution. Similarly, small $\epsilon$ values were observed in ab initio calculated charge distribution in benzoylacetone (Schiøtt et al., 1998), but not in the experimental analysis by Madsen et al. (1998). It should also be noted that the ellipticity, as a ratio of second derivatives, is very sensitive to small differences in the model and therefore it is difficult to obtain an accurate value (Pérès et al., 1999).

The Laplacian value for the $\mathrm{O} 3-\mathrm{H} 3$ bond $\mathrm{CP}\left(-34.9\right.$ e $\AA^{-5}$ is the highest for all the bonds in the structure and seems to be typical. Similar or higher values were reported for example in glycyl-L-threonine dihydrate (-48.0 e $\AA^{-5}$; Benabicha et al., 2000), 2,2'-diethynylenedibenzoic acid (-37.7 e $\AA^{-5}$; Smith et al., 2001) and in citrinin (-33.4 e $\AA^{-5}$ and -49.8 e $\AA^{-5}$; Roversi et al., 1996). These high values might be related to the large asymmetry of the $\mathrm{CP}$ position on the bond. It lies so close to the $\mathrm{H}$ atom that it falls within the valence-shell charge concentration (VSCC). 


\section{Table 10}

Net atomic charges derived from monopole population $q\left(P_{\text {val }}\right)$ and from AIM topological analysis $q(\Omega)$ in the MCPD molecule for the two multipolar models.

$q\left(P_{\text {val }}\right)=N_{\text {val }}-P_{\text {val }}$, where $N_{\text {val }}$ is the number of valence electrons for the neutral atoms. $\Omega$ is the atomic volume

\begin{tabular}{lllllr}
\hline Model & EXP_MUL & EXP_MUL & THEO_MUL & THEO_MUL & EXP_MUL \\
atom & $q\left(P_{\text {val }}\right)(\mathrm{e})$ & $q(\Omega)(\mathrm{e})$ & $q\left(P_{\text {val }}\right)(\mathrm{e})$ & $q(\Omega)(\mathrm{e})$ & $\Omega\left(\AA^{3}\right)$ \\
\hline O1 & -0.30 & -1.09 & -0.32 & -1.14 & 27.2 \\
O3 & -0.29 & -1.26 & -0.21 & -1.21 & 15.8 \\
C1 & +0.06 & +0.87 & +0.02 & +0.86 & 8.9 \\
C2 & +0.32 & +0.10 & -0.05 & -0.03 & 9.7 \\
C21 & -0.65 & -0.23 & -0.08 & +0.14 & 10.5 \\
C3 & -0.09 & +0.67 & -0.03 & +0.53 & 7.0 \\
C4 & -0.32 & -0.19 & -0.01 & +0.09 & 10.9 \\
C5 & -0.32 & -0.11 & -0.16 & +0.04 & 10.5 \\
H3 & +0.35 & +0.66 & +0.20 & +0.65 & 1.5 \\
H4 & +0.16 & +0.08 & +0.08 & +0.02 & 8.7 \\
H5 & +0.16 & +0.07 & +0.11 & +0.01 & 10.9 \\
H21 $A$ & +0.21 & +0.10 & +0.14 & +0.04 & 6.7 \\
H21B & +0.21 & +0.10 & +0.05 & -0.02 & 14.1 \\
\hline & & & & &
\end{tabular}

\subsection{Strong and weak hydrogen bonds}

The atoms in molecules method (Bader, 1990) is an essential tool for analyzing intermolecular interactions, especially hydrogen bonds. In the MCPD crystal, the critical point connected with the strongest $\mathrm{O} 3-\mathrm{H} 3 \cdots \mathrm{O} 1$ hydrogen bond is clearly seen (Fig. 10b) and its characteristics are consistent with its classification as a 'strong' hydrogen bond.

The RAHB effect is of course much more pronounced for intramolecular hydrogen bonds. In the case of benzoylacetone (Madsen et al., 1998), the electron density $\rho$ at the CP was as high as $0.89 \mathrm{e} \AA^{-3}$. The negative Laplacian value testified for the covalent contribution in this hydrogen bond and the vibration coordinate $q$ was $0.016 \AA$ and showed almost full delocalization.

In MCPD the hydrogen bond is intermolecular and therefore the effect of RAHB is less striking. However, the $\rho$ value at the $\mathrm{H} 3 \cdots \mathrm{O} 1 \mathrm{CP}, 0.27 \mathrm{e} \AA^{-3}$, is high and similar to those reported for intermolecular hydrogen bonds in 2,2'-diethynylenedibenzoic acid (Smith et al., 2001) and in a chargeassisted hydrogen bond in potassium hydrogen (+)-tartrate (Koritsanszky et al., 2000). The positive value of the Laplacian points towards a 'charge depletion' at the critical point and is in agreement with the 'separated charge' description of the hydrogen bond. There is no indication of covalent contribution, but the $\mathrm{O} 3-\mathrm{H} 3$ bond $\mathrm{CP}$ displays a large negative Laplacian value. Also, the topological charges within the hydrogen bond are in agreement with the RAHB model as modeled by Madsen et al. (1998). The topological charges on both $\mathrm{O}$ atoms are highly negative and have almost equal values, while the $\mathrm{H}$ atoms bear substantial positive charge ( $c f$. Table 10).

In the crystal structure there are some weak $\mathrm{C}-\mathrm{H} \cdots \mathrm{O}$ contacts, as listed in Table 11. According to the theory of atoms in molecules, such a contact might be regarded as a hydrogen bond if, among other conditions defined by Koch \& Popelier (1995), there is a critical point along the $\mathrm{H} \cdots \mathrm{O}$ path.
Some authors (Steiner, 1999; Steiner \& Desiraju, 1999) differentiated weak hydrogen bonds from van der Waals contacts, mainly on the basis of directionality of the contact. The MCPD structure turned out to be a convenient object to study these differences. There are four weak intermolecular $\mathrm{C}-\mathrm{H} \cdots \mathrm{O}$ contacts with $\mathrm{H} \cdots \mathrm{O}$ distances shorter than $2.8 \AA$ (Table 11). The shortest one, $\mathrm{C} 4-\mathrm{H} 4 \cdots \mathrm{O} 1$, has a very unfavourable $\mathrm{C}-\mathrm{H} \cdots \mathrm{O}$ angle, $93.5^{\circ}$, and the topological maps show a bonding path, but with high ellipticity. This can be regarded as an additional argument to consider it as a mere van der Waals contact.

The next two contacts, $\mathrm{C} 21-\mathrm{H} 21 A \cdots \mathrm{O} 3(-x, y,-z+1)$ and $\mathrm{C} 21-\mathrm{H} 21 B \cdots \mathrm{O} 1 \quad\left(-x+\frac{1}{2},-y+\frac{1}{2},-z+2\right)$ can be treated as very weak hydrogen bonds as the $\mathrm{C}-\mathrm{H} \cdots \mathrm{O}$ angles are more favourable (134.0 and $130.1^{\circ}$, respectively). These are quite typical values for this kind of weak interaction (e.g. Desiraju, 1996; Steiner, 2002) and the CPs do exist. Furthermore, for the $\mathrm{C} 21-\mathrm{H} 21 A \cdots \mathrm{O} 3$ hydrogen bond it can be seen that the $\mathrm{H}$ atom points towards the concentration of charge in the VSCC of O3.

For the longest interaction $\mathrm{C} 4-\mathrm{H} 4 \cdots \mathrm{O} 3$ $\left(-x+\frac{1}{2},-y+\frac{1}{2},-z+1\right)$ with a $D-\mathrm{H} \cdots A$ angle of $111.3^{\circ}$, the charge-density distribution does not display any critical point nor a bond path between the $\mathrm{H} 4$ and $\mathrm{O} 3$ atoms. Instead, the $\mathrm{CP}$ search in this region leads to a bond path between $\mathrm{C} 4$ and $\mathrm{O} 1$ atoms.

Charge-density topology and $\mathrm{C}-\mathrm{H} \cdots \mathrm{O}$ angles allow us to consider two of the $\mathrm{C}-\mathrm{H} \cdots \mathrm{O}$ contacts in the MCPD structure as hydrogen bonds. These findings, as well as the importance of the angular geometry of hydrogen bonds agree well with the theoretical and experimental study of Gatti et al. (2002) on the fundamental properties and nature of $\mathrm{C}-\mathrm{H} \cdots \mathrm{O}$ interactions in the crystal of 3,4-bis(dimethylamino)-3-cyclobutene-1,2dione.

\subsection{Atomic charges}

An electroneutrality constraint was applied when the $P_{\text {val }}$ and $P_{\text {vir }}$ variables were refined. The electroneutrality constraint implemented in MoPro can be readily applied to the VIR modelling without modification of the software. For instance, when the conjugate gradient method is used, one $P_{\text {val }}$ or $P_{\text {vir }}$ variable is not refined and its shift is set to the opposite of the sum of the other shifts. The $P_{\text {vir }}$ populations are expected to remain positive, which turned out to be the case for all virtual atoms in both EXP_MUL and THEO_MUL refinements of MCPD. As the virtual atoms have all positive $P_{\text {vir }}$ populations, to maintain electroneutrality the $P_{\text {val }}$ valence populations of the real atoms are globally lower in the VIR models compared with the multipolar atom model.

The atomic charges of the EXP_MUL and THEO_MUL models were determined from the monopole population $\left(P_{\text {val }}\right)$ and the AIM analysis (Table 10). The AIM topological charges $q(\Omega)$ and the atomic volumes of all atoms were calculated from the charge integration of electron distribution within the atomic basins, using the VMoPro code. 


\section{Table 11}

Topological properties of the electron density for the intermolecular contacts in the MPCD crystal (see Table 9 for legend).

\begin{tabular}{llllllllll}
\hline Hydrogen bond & $d_{12}$ & $d_{1 \mathrm{cp}}$ & $d_{2 \mathrm{cp}}$ & $\rho\left(\mathbf{r}_{\mathrm{cp}}\right)$ & $\nabla^{2} \rho\left(\mathbf{r}_{\mathrm{cp}}\right)$ & $\lambda_{1}$ & $\lambda_{2}$ & $\lambda_{3}$ & $\epsilon$ \\
\hline $\mathrm{O} 1 \cdots \mathrm{H} 3^{\mathrm{i}}$ & 1.5264 & 1.0887 & 0.4537 & 0.2666 & 6.69 & -1.34 & -1.07 & 9.11 & 0.25 \\
$\mathrm{O} 1 \cdots \mathrm{H} 4^{\mathrm{i}}$ & 2.6754 & 1.4207 & 1.4178 & 0.0642 & 0.98 & -0.19 & -0.07 & 1.25 & 1.57 \\
$\mathrm{O} 3 \cdots \mathrm{H} 21 A^{\text {ii }}$ & 2.7383 & 1.5427 & 1.1967 & 0.0318 & 0.46 & -0.10 & -0.09 & 0.64 & 0.02 \\
$\mathrm{O} 1 \cdots \mathrm{H} 21 B^{\text {ii }}$ & 2.7446 & 1.5798 & 1.1676 & 0.0325 & 0.44 & -0.11 & -0.08 & 0.63 & 0.42 \\
$\mathrm{O} 3 \cdots \mathrm{H} 4^{\text {iv }}$ & 2.7874 & - & - & - & - & - & - & - & - \\
\hline
\end{tabular}

Symmetry codes: (i) $x, y, z-1$; (ii) $-x,-y,-z+1$; (iii) $-x+\frac{1}{2},-y+\frac{1}{2},-z+2$; (iv) $-x+\frac{1}{2},-y+\frac{1}{2},-z+1$.

interacting dimers in the crystal were estimated and agree generally well between the four models. For MCPD, the experimental models show a significant reduction of the electrostatic energy compared with the theoretical models. In addition, $E_{\mathrm{es}}$ is also slightly attenuated in the VIR models compared with the MUL models.

MK is grateful to Université de Lorraine for providing him with 1 month of invited professorship. The CNRS is thanked for a

For both sets of $q\left(P_{\mathrm{val}}\right)$ charges, the $\mathrm{C}$ atoms connected to the $\mathrm{O}$ atoms are positively charged, while those related to hydrogen are negatively charged, consistent with the electronegativity of these atoms $(\mathrm{O}>\mathrm{C}>\mathrm{H})$. Negative $q(\Omega)$ charges are found on both $\mathrm{O}$ atoms and positive charges on the oxygen-bound $\mathrm{C} 1$ and $\mathrm{C} 3$ atoms.

As usual, due to the very different charge definitions, a large discrepancy is observed for the polar groups $(\mathrm{C}=\mathrm{O}$ and $\mathrm{C}-$ $\mathrm{O}-\mathrm{H})$ between the $P_{\text {val }}$ derived charges and the topological integrated charges, the latter showing larger magnitudes. The largest charge difference between $q\left(P_{\text {val }}\right)$ and $q(\Omega)$ is observed for the hydroxyl $\mathrm{O} 3$ atom and is as high as 1.0 for both multipolar models. The $q$ (AIM) charges of the $\mathrm{O}$ atoms are highly negative, lower than -1.0 , for the two multipolar models.

The $q\left(P_{\mathrm{val}}\right)$ positive charge of the hydroxyl $\mathrm{H}$ atom is larger than those of the carbon-bound $\mathrm{H}$ atoms but takes only about half the $q(\Omega)$ value. The integrated charge of $\mathrm{H} 3(+0.66 \mathrm{e})$ strongly confirms the very acidic character of the $\mathrm{H} 3$ atom (a large positive charge and a small atomic basin volume). The chemical significance of topological charges (e.g. Kubicki et al., 2002) is confirmed.

\section{Conclusion}

The electron-density distribution in the crystals of 2-methyl1,3-cyclopentanedione was determined from synchrotron $\mathrm{X}$ ray diffraction data at ultra-high resolution. The structure was analysed in terms of its geometry, molecular packing and intraand intermolecular interactions. The charge-density analysis of the MCPD crystal confirms the resonance within the $\mathrm{O}=\mathrm{C}-\mathrm{C}=\mathrm{C}-\mathrm{OH}$ fragment. This resonance in turn, by the RAHB effect, strengthens the intermolecular $\mathrm{O}-\mathrm{H} \cdots \mathrm{O}$ hydrogen bond, but this effect is far less evident than for the intramolecular resonance-assisted hydrogen bonds. The electrostatic character of this hydrogen bond appears to be dominant. The distribution of dipole moments within the crystals is responsible for the unusual stability of MCPD crystals.

The multipolar atom model shows more flexibility than the VIR model to reproduce the deformation electron density features of the molecule. The four electron density models (EXP-MUL, EXP-VIR, THEO-MUL and THEO-VIR), however, exhibit well correlated ESPs. The electrostatic interaction energies computed for the different molecular postdoctoral grant to AN. The authors thank Professor Mohamed Souhassou for fruitful discussions.

\section{References}

Afonine, P. V., Grosse-Kunstleve, R. W., Adams, P. D., Lunin, V. Y. \& Urzhumtsev, A. (2007). Acta Cryst. D63, 1194-1197.

Afonine, P. V., Lunin, V. Y., Muzet, N. \& Urzhumtsev, A. (2004). Acta Cryst. D60, 260-274.

Ahmed, M., Jelsch, C., Guillot, B., Lecomte, C. \& Domagała, S. (2013). Cryst. Growth Des. 13, 315-325.

Allen, F. H. (1986). Acta Cryst. B42, 515-522.

Bader, R. F. W. (1990). Atoms in Molecules: a Quantum Theory. Oxford: Clarendon Press.

Bąk, J. M., Domagała, S., Hübschle, C., Jelsch, C., Dittrich, B. \& Dominiak, P. M. (2011). Acta Cryst. A67, 141-153.

Becke, A. D. (1993). J. Chem. Phys. 98, 5642-5648.

Benabicha, F., Pichon-Pesme, V., Jelsch, C., Lecomte, C. \& Khmou, A. (2000). Acta Cryst. B56, 155-165.

Bertolasi, V., Ferretti, V., Gilli, P., Gilli, G., Issa, Y. M. \& Sherif, O. E. (1993). J. Chem. Soc. Perkin Trans. 2, pp. 2223-2228.

Bertolasi, V., Gilli, P., Ferretti, V. \& Gilli, G. (1991). J. Am. Chem. Soc. 113, 4917-4925.

Bertolasi, V., Gilli, P., Ferretti, V. \& Gilli, G. (1998). Acta Cryst. B54, $50-65$.

Bertolasi, V., Gilli, P., Ferretti, V., Gilli, G. \& Fernàndez-Castaño, C. (1999). Acta Cryst. B55, 985-993.

Bertolasi, V., Gilli, P., Ferretti, V., Gilli, G., Vaughan, K. \& Jollimore, J. V. (1999). Acta Cryst. B55, 994-1004.

Bruker (2004). APEX2, SADABS, SAINT and XPREP. Bruker AXS Inc., Madison, Wisconsin, USA.

Cheeseman, J. R., Carroll, M. T. \& Bader, R. F. W. (1988). Chem. Phys. Lett. 143, 450-458.

Coppens, P., Guru Row, T. N., Leung, P., Stevens, E. D., Becker, P. J. \& Yang, Y. W. (1979). Acta Cryst. A35, 63-72.

Dadda, N., Nassour, A., Guillot, B., Benali-Cherif, N. \& Jelsch, C. (2012). Acta Cryst. A68, 452-463.

Dahaoui, S., Jelsch, C., Howard, J. A. K. \& Lecomte, C. (1999). Acta Cryst. B55, 226-230.

Desiraju, G. R. (1996). Acc. Chem. Res. 29, 441-449.

Dovesi, R., Saunders, V. R., Roetti, C., Orlando, R., Zocovich-Wilson, C. M., Pascale, F., Civalleri, B., Doll, K., Harrison, N. M., Bush, I. J., D'Arco, Ph. \& Llunell, M. (2010). CRYSTAL09 User's Manual. University of Turin, Italy.

Espinosa, E., Lecomte, C., Ghermani, N. E., Devémy, J., Rohmer, M. M., Bénard, M. \& Molins, E. (1996). J. Am. Chem. Soc. 118, 25012502.

Etter, M. C., Urbanczyk-Lipkowska, Z. \& Jahn, D. A. (1986). J. Am. Chem. Soc. 108, 5871-5876.

Frisch, M. J. et al. (2009). GAUSSIAN09. Revision A.02. Gaussian Inc., Wallingford, CT, USA.

Gatti, C., May, E., Destro, R. \& Cargnoni, F. (2002). J. Phys. Chem. A, 106, 2707-2720. 
Gilli, G., Bellucci, F., Ferretti, V. \& Bertolasi, V. (1989). J. Am. Chem. Soc. 111, 1023-1028.

Gilli, G., Bertolasi, V., Ferretti, V. \& Gilli, P. (1993). Acta Cryst. B49, 564-576.

Gilli, P., Bertolasi, V., Ferretti, V. \& Gilli, G. (2000). J. Am. Chem. Soc. 122, 10405-10417.

Guillot, B. (2011). Acta Cryst. A67, C511-C512.

Guillot, R., Muzet, N., Dahaoui, S., Lecomte, C. \& Jelsch, C. (2001). Acta Cryst. B57, 567-578.

Guillot, B., Viry, L., Guillot, R., Lecomte, C. \& Jelsch, C. (2001). J. Appl. Cryst. 34, 214-223.

Hansen, N. K. \& Coppens, P. (1978). Acta Cryst. A34, 909-921.

Hariharan, P. C. \& Pople, J. A. (1973). Theor. Chim. Acta, 28, 213222.

Hirshfeld, F. L. (1976). Acta Cryst. A32, 239-244.

Hohenberg, P. \& Kohn, W. (1964). Phys. Rev. B, 136, 864-871.

Jeffrey, G. A. \& Saenger, W. (1991). Hydrogen Bond in Biological Structures, Ch. 2, p. 37. Berlin: Springer.

Jelsch, C., Guillot, B., Lagoutte, A. \& Lecomte, C. (2005). J. Appl. Cryst. 38, 38-54.

Katrusiak, A. (1989). Acta Cryst. C45, 1897-1899.

Katrusiak, A. (1990a). Acta Cryst. C46, 1289-1293.

Katrusiak, A. (1990b). Acta Cryst. B46, 246-256.

Katrusiak, A. (1991). High Pressure Res. 6, 155-167.

Katrusiak, A. (2001). J. Mol. Graph. Model. 19, 363-367.

Kissel, L., Zhou, B., Roy, S. C., Sen Gupta, S. K. \& Pratt, R. H. (1995). Acta Cryst. A51, 271-288.

Klooster, W. T., Swaminathan, S., Nanni, R. \& Craven, B. M. (1992). Acta Cryst. B48, 217-227.

Koch, U. \& Popelier, P. L. A. (1995). J. Phys. Chem. 99, 9747-9754.

Koritsanszky, T., Zobel, D. \& Luger, P. (2000). J. Phys. Chem. A, 104, 1549-1556.

Kubicki, M., Borowiak, T., Dutkiewicz, G., Souhassou, M., Jelsch, C. \& Lecomte, C. (2002). J. Phys. Chem. B, 106, 3706-3714.

Le Page, Y. \& Gabe, E. J. (1979). J. Appl. Cryst. 12, 464-466.

Lee, C., Yang, W. \& Parr, R. G. (1988). Phys. Rev. B, 37, 785-789.

Madsen, A. Ø. (2006). J. Appl. Cryst. 39, 757-758.

Madsen, G. K. H., Iversen, B. B., Larsen, F. K., Kapon, M., Reisner, G. M. \& Herbstein, F. H. (1998). J. Am. Chem. Soc. 120, 1004010045.

Meindl, K. \& Henn, J. (2008). Acta Cryst. A64, 404-418.

Møller, C. \& Plesset, M. S. (1934). Phys. Rev. 46, 618-622.
Mullen, D. \& Hellner, E. (1977). Acta Cryst. B33, 3816-3822.

Munshi, P., Jelsch, C., Hathwar, V. R. \& Row, T. N. G. (2010). Cryst. Growth Res. 10, 1516-1526

Parrish, D., Zhurova, E. A., Kirschbaum, K. \& Pinkerton, A. A. (2006). J. Phys. Chem. B, 110, 26442-26447.

Pérès, N., Boukhris, A., Souhassou, M., Gavoille, G. \& Lecomte, C. (1999). Acta Cryst. A55, 1038-1048.

Pichon-Pesme, V., Lecomte, C., Wiest, R. \& Benard, M. (1992). J. Am. Chem. Soc. 114, 2713-2715.

Poulain-Paul, A., Nassour, A., Jelsch, C., Guillot, B., Kubicki, M. \& Lecomte, C. (2012). Acta Cryst. A68, 715-728.

Roversi, P., Merati, F., Destro, R. \& Barzaghi, M. (1996). Can. J. Chem. 74, 1145-1161.

Scheringer, C. (1980). Acta Cryst. A36, 205-210.

Scheringer, C., Kutoglu, A., Mullen, D. \& Hellner, E. (1978). Acta Cryst. A34, 475-476.

Schiøtt, B., Iversen, B. B., Hellerup Madsen, G. K. \& Bruice, T. C. (1998). J. Am. Chem. Soc. 120, 12117-12124.

Semmingsen, D. (1974). Acta Chem. Scand. Ser. B, 28, 169-174.

Semmingsen, D., Hollander, F. J. \& Koetzle, T. F. (1977). J. Chem. Phys. 66, 4405-4412.

Sheldrick, G. M. (2008). Acta Cryst. A64, 112-122.

Singh, I. \& Calvo, C. (1975). Can. J. Chem. 53, 1046-1050.

Smith, G. T., Howard, J. A. K. \& Wallis, J. D. (2001). Phys. Chem. Chem. Phys. 3, 4501-4507.

Steiner, T. (1999). Chem. Commun. pp. 313-314.

Steiner, T. (2002). Angew. Chem. Int. Ed. 41, 48-76.

Steiner, T. \& Desiraju, G. R. (1999). Chem. Commun. pp. 891892.

Stewart, R. F. (1976). Acta Cryst. A32, 565-574.

Su, Z. \& Coppens, P. (1998). Acta Cryst. A54, 646-652.

Volkov, A., Abramov, Y. A. \& Coppens, P. (2001). Acta Cryst. A57, 272-282.

Wu, G., Rodrigues, B. L. \& Coppens, P. (2002). J. Appl. Cryst. 35, 356359.

Yearley, E. J., Zhurova, E. A., Zhurov, V. V. \& Pinkerton, A. A. (2008). J. Mol. Struct. 890, 240-248.

Yukhnevich, G. V. (2009). Crystallogr. Rep. 54, 184-189.

Zhurova, E. A., Matta, C. F., Wu, N., Zhurov, V. V. \& Pinkerton, A. A. (2006). J. Am. Chem. Soc. 128, 8849-8861.

Zhurov, V. V., Zhurova, E. A. \& Pinkerton, A. A. (2008). J. Appl. Cryst. 41, 340-349. 